\title{
Crust and upper-mantle seismic anisotropy variations from the coast to inland in central and Southern Mexico
}

\author{
Jorge, Castellanos, ${ }^{1, *}$ Xyoli Pérez-Campos, ${ }^{2}$ Raúl Valenzuela, ${ }^{2}$ Allen Husker ${ }^{2}$ \\ and Luca Ferrari ${ }^{3}$ \\ ${ }^{1}$ Posgrado en Ciencias de la Tierra, Universidad Nacional Autónoma de México, Mexico City 04510, Mexico \\ ${ }^{2}$ Departamento de Sismología, Instituto de Geofisica, Universidad Nacional Autónoma de México, Mexico City 04510, Mexico. \\ E-mail:xyoli@geofisica.unam.mx \\ ${ }^{3}$ Centro de Geociencias, Universidad Nacional Autónoma de México, Campus Juriquilla, 76230 Queretaro, Qro., Mexico
}

Accepted 2017 April 26. Received 2017 April 24; in original form 2015 October 4

\begin{abstract}
S U M M A R Y
Subduction zones are among the most dynamic tectonic environments on Earth. Deformation mechanisms of various scales produce networks of oriented structures and faulting systems that result in a highly anisotropic medium for seismic wave propagation. In this study, we combine shear wave splitting inferred from receiver functions and the results from a previous $S K S$-wave study to quantify and constrain the vertically averaged shear wave splitting at different depths along the 100-station MesoAmerican Subduction Experiment array. This produces a transect that runs perpendicular to the trench across the flat slab portion of the subduction zone below central and southern Mexico. Strong anisotropy in the continental crust is found below the Trans-Mexican Volcanic Belt (TMVB) and above the source region of slow-slip events. We interpret this as the result of fluid/melt ascent. The upper oceanic crust and the overlying lowvelocity zone exhibit highly complex anisotropy, while the oceanic lower crust is relatively homogeneous. Regions of strong oceanic crust anisotropy correlate with previously found low $V_{p} / V_{s}$ regions, indicating that the relatively high $V_{s}$ is an anisotropic effect. Upper-mantle anisotropy in the southern part of the array is in trench-perpendicular direction, consistent with the alignment of type-A olivine and with entrained subslab flow. The fast polarization direction of mantle anisotropy changes to $\mathrm{N}-\mathrm{S}$ in the north, likely reflecting mantle wedge corner flow perpendicular to the TMVB.
\end{abstract}

Key words: North America; Seismic anisotropy; Subduction zone processes.

\section{INTRODUCTION}

The geometry of the Cocos plate subducting beneath the North America plate is complex and varies along the Middle America Trench (MAT). Singh \& Pardo (1993) first suggested that the slab becomes subhorizontal under Central Mexico. Pardo \& Suárez (1995) mapped the change on the slab's dip angle using local earthquake locations. Recently, more precise estimations of this geometry were obtained using teleseismic converted phases (Pérez-Campos et al. 2008; Kim et al. 2010, 2012; Song \& Kim 2012a,b) and seismic wave tomography (Husker \& Davis 2009; Chen \& Clayton 2012) using an array of 100 broad-band temporary stations known as the MesoAmerican Subduction Experiment (MASE). MASE crossed Mexico from Acapulco, Guerrero, at the Pacific coast, to Tempoal,

* Now at: Seismological Laboratory, California Institute of Technology, Pasadena, CA 91125, USA.
Veracruz, at the Gulf of Mexico (Fig. 1). Pérez-Campos et al. (2008) showed that the subducting Cocos plate beneath central Mexico is flat, and lies just below the base of the continental crust over a distance of $250 \mathrm{~km}$ from the coast before plunging steeply into the mantle just south of Mexico City.

Another feature of the Mexican subduction zone is the presence of a thin, low-velocity layer (LVL) observed above the entire length of the horizontal segment of the subducted plate (Pérez-Campos et al. 2008). This layer extends parallel to the trench up to where the Cocos plate changes its geometry, to a dip of $41^{\circ}$ to the north (Dougherty et al. 2012) and to a dip of $26^{\circ}$ (Melgar \& Pérez-Campos 2011 ) to the south (Dougherty \& Clayton 2014). Based on the null seismicity at the interface in this region and the tensional stress regime in the upper plate (Singh \& Pardo 1993), this layer has been proposed to absorb most of the deformation between the subducting and overlying plates, which would explain the horizontal geometry without significant coupling (Kim et al. 2010). It has been suggested that this LVL has a high pore pressure that is confined by 


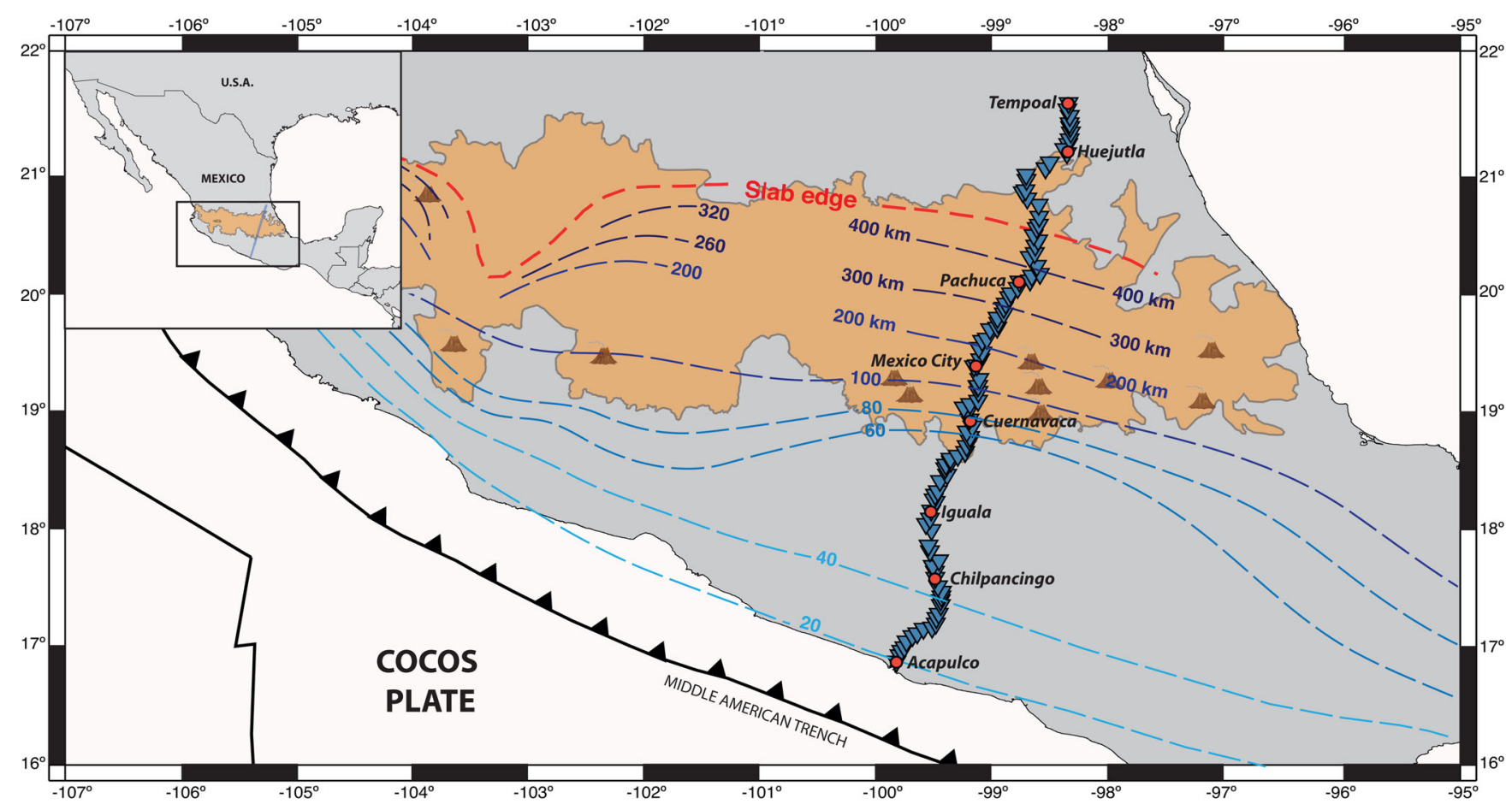

Figure 1. Location of the broad-band seismic stations used in the MASE array (blue inverted triangles). The orange area represents the Trans-Mexican Volcanic Belt. Brown triangles show location of active volcanoes. Slab isodepth contours are from the compilation by Ferrari et al. (2012). Main cities along the array are marked with red circles.

a low permeability layer directly above the interface in the continental lower crust (Song et al. 2009; Kim et al. 2010), and that it might be composed of mechanically weak hydrated minerals, such as talc (Kim et al. 2010, 2013). The latter is in accordance with numerical experiments of Manea et al. (2013), which model the LVL as a remnant of the mantle wedge that experienced significant serpentinization since the onset of flat subduction. To further constrain how the upper and lower plates deform and mantle flows in response the slab flattening, we study here the seismic anisotropy along MASE array.

In any anisotropic material, seismic waves travel at different velocities depending on their direction of propagation and polarization. Such variation can produce a complex effect on the wave field in which an $S$ wave is separated into two orthogonal phases with different velocity and polarization. These phases, one traveling faster than the other, progressively split in time as they propagate along the anisotropic material and exhibit a time delay $(\delta t)$. Two different arrivals are then observed on a recorded seismogram. Various studies have revealed the existence of seismic anisotropy along the MASE array (e.g. Rojo Garibaldi 2011; Ponce Cortés 2012; Stubailo et al. 2012; Song \& Kim 2012a,b; van Benthem et al. 2013). However, the low degree of resolution of the techniques used hampers the crustal versus mantle anisotropy discrimination needed to properly characterize crustal stress and the mantle flow direction. Therefore, we examined the shear wave splitting observed in $P$ to $S$ converted phases in the form of receiver functions (RFs) generated at different interfaces with strong impedance contrasts to quantify and constrain the seismic anisotropy along the MASE array (MASE 2007; Fig. 1). Our method of analysis allowed us to assess the vertically averaged shear wave anisotropy exclusively contained in the continental crust and oceanic slab in terms of a fast polarization direction, $\varphi$, and a time delay, $\delta t$. We then used these parameters to remove their contribution from the anisotropy determined by a previous $S K S$ study
(Rojo Garibaldi 2011) along the same array of seismic stations to determine the anisotropy corresponding to the upper mantle along the MASE array.

\section{PREVIOUS RECEIVER FUNCTION OBSERVATIONS}

Teleseismic waveform modeling, or RF analysis (Langston 1977), is widely used in seismic structure studies (e.g. Vinnik et al. 1997; Sandvol et al. 1998). This technique uses converted $P$ to $S$ teleseismic waves, or $P s$ waves, to map structural features beneath a seismic station. The $P S$ wave is generated when an incident $P$ wave encounters an acoustic impedance contrast in the medium. The amplitudes observed in these time-series are directly related to the incidence angle of the $P$ wave and the contrast of velocities in the medium. The timing of the arrivals depends on the depth of the discontinuity, the $P$ - and $S$-wave velocities of the layers and the ray parameter of the incoming wave. In practice, it is non-trivial to observe the $P s$ arrival in regular seismograms, since it is usually obscured by the $P$-wave coda. However, it is possible to isolate the converted $P s$ wave, and therefore, the structure response beneath the station by deconvolving the $P$ pulse from the rest of the seismogram (e.g. Langston 1979; Ammon 1991).

We use RFs for 149 teleseismic events $\left(30^{\circ} \leq \Delta \leq 90^{\circ} ; M_{\mathrm{w}} \geq\right.$ 5.8) recorded along the MASE array and obtained by Pérez-Campos et al. (2008). They windowed the signals $30 \mathrm{~s}$ before the $P$-wave arrival and $90 \mathrm{~s}$ after, with a 5 per cent cosine taper. Then, they deconvolved the vertical component from the horizontal components in the frequency domain. A water level of $0.0001,0.001,0.01$ and 0.1 was first applied to the spectra of the horizontal components to avoid division by zero and enhancement of noise (Clayton \& Wiggins 1976). RFs were filtered with a Gaussian filter before 
(a)
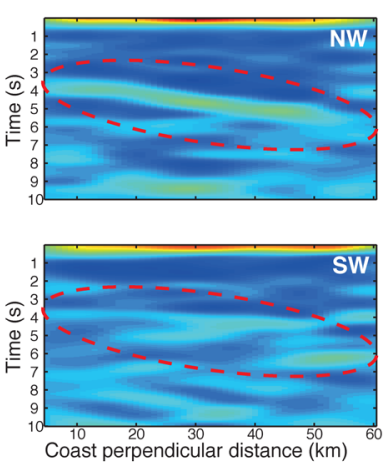

(b)

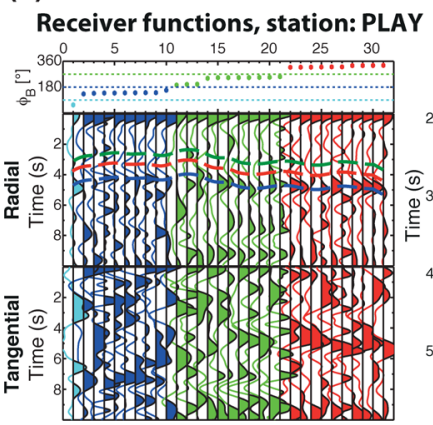

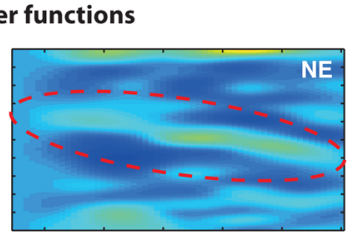

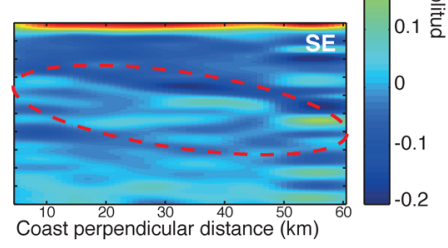

Coast perpendicular distance $\stackrel{50}{20}(\mathrm{~km})$
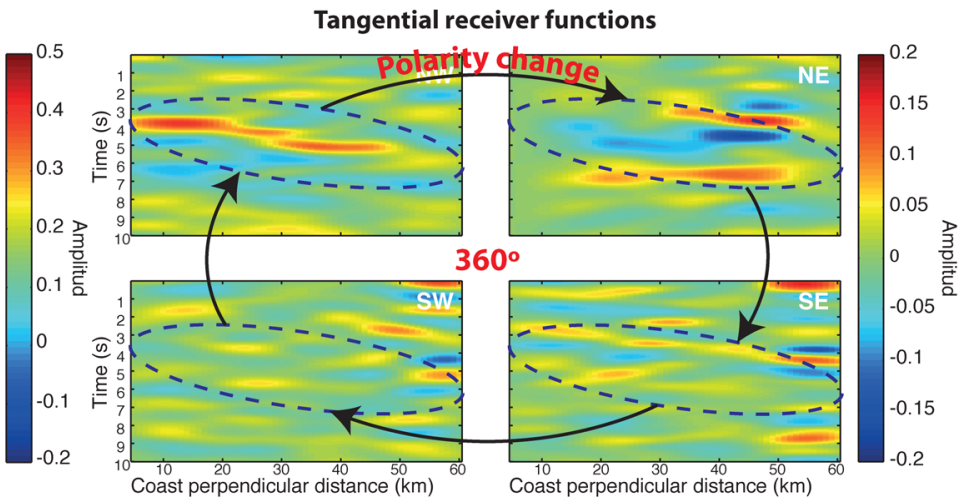
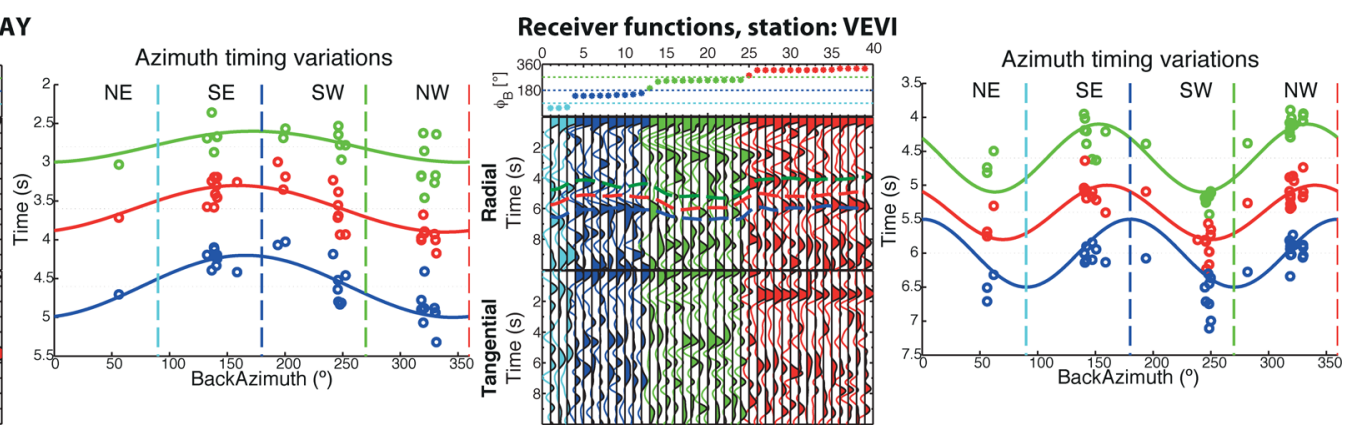

Figure 2. (a) Stacked radial and tangential RF profile for each backazimuth quadrant, for the first $60 \mathrm{~km}$ of MASE. The image shows the slab interface dipping deeper as the distance from the coast increases. Radial RFs arriving from the northern quadrants appear to be slightly more energetic and coherent than those arriving from the southern quadrants. Tangential RFs show a distinctive amplitude reversal with a $360^{\circ}$ periodicity that is characteristic of dipping layers. (b) $\mathrm{RF}$ timing variations of a station located in the first dipping region of the slab (left) and another one located in the subhorizontal region of the slab (right). The colour of the RFs indicates its backazimuth quadrant (light blue is NE, blue is SE, green is SW and red is NW). The green curves represent the Ps phase periodicity for the continental crust, whereas the red and blue curves represent it for the upper and lower oceanic crusts, respectively.

transformation back into the time domain. The width of the used Gaussian filter was either 2.5 or 5 .

Preliminary analysis done by Greene Gondi (2009) on those RFs revealed significant energy content in the tangential RFs, as well as azimuthal variations in the $P S$-wave amplitude, timing and polarity for both, radial and tangential RFs. These particular features indicate a departure from an Earth consisting of flat homogeneous isotropic layers, since such an Earth would produce no tangential energy and would be free of such azimuthal variations. Fig. 2(a) displays RF profiles, grouped by backazimuth quadrant, along the southernmost $60 \mathrm{~km}$ of the MASE experiment, where the dip of the slab has been established to be $\sim 19^{\circ}$ (Pérez-Campos et al. 2008). The image shows the slab interface with a negative pulse over a distinctive positive pulse, dipping down as the distance from the coast grows. In addition, there is significant azimuth variation in the mapped interface geometry.

In the radial RFs, $P s$ waves arriving from the northern geographic quadrants (downdip) appear to be more energetic and coherent than those arriving from the southern quadrants (updip). Moreover, the amplitudes of direct $P$ waves are larger for azimuths within the NW and SE quadrants, which are approximately coincident with the strike of the subducting slab. Tangential RFs, on the other hand, appear to be even more complex. Aside from significant energy content and variation from all geographic quadrants, $P s$ waves exhibit an amplitude reversal with a $360^{\circ}$ periodicity, in agreement with the effect of dipping layers (Cassidy 1992). A similar analysis conducted at each station of the MASE array also revealed azimuthal variations and substantial energy content in both, radial and tangential, RFs. However, the lack of observed consistency in the maximum amplitude direction between stations and the additional existence of 'split' $P s$ phases led us to consider the presence of seismic anisotropy, since dipping layers alone cannot produce these effects.

The expected effect of anisotropy is that $P S$ amplitude of the tangential component is minimum along the fast and slow polarization directions and reaches a maximum $45^{\circ}$ from either one of these axes (Bowman \& Ando 1987; Silver \& Chan 1988; Savage et al. 1990; Levin \& Park 1998). Moreover, in transversely anisotropic layers with horizontal symmetry axes, RFs tend to display a $180^{\circ}$ periodicity as a function of backazimuth for the arrival times of the $P S$ phases, as well as a change in polarity in its tangential component whenever there is a change from an azimuth of fast velocity to one of slow. This is in contrast to dipping symmetry axes, which have waveforms with $360^{\circ}$ periodicity (Savage 1998). Fig. 2(b) shows a comparison between the RFs' timing variation of two stations, one located in the first $60 \mathrm{~km}$ of the array, and another one at $\sim 110 \mathrm{~km}$ from the coast, where the slab has been found to be horizontal (Pérez-Campos et al. 2008). Stations located above the dipping part of the slab clearly show $360^{\circ}$ periodicity, while those above regions of plane layered high anisotropy show $180^{\circ}$ periodicity (Fig. 2b).

\section{ESTIMATION OF ANISOTROPY PARAMETERS}

The first step to estimate the anisotropy was to remove the RF variations due to the backazimuth and ray parameter of the incoming rays. To remove this effect, we performed a normal moveout 
correction that brings the conversion point of the $P s$ phases of interest to one in common. This procedure was analogous to relocating the source of every RF to the same depth and distance from the station, leaving only the effect due to azimuth. In this study, we used a slight modification of the 'Four-Pin' moveout correction (Chen \& Niu 2013), which approximated the ray parameter of the primary $P$ s phase with the direct $P$ wave (e.g. Chevrot \& van der Hilst 2000; Zhu \& Kanamori 2000; Nair et al. 2006). We assumed the velocity model by Iglesias et al. (2010), the geometry of the slab reported by Pérez-Campos et al. (2008) and a reference ray parameter of $6.5 \mathrm{~s}$ per degree to match the $P s$ arrivals for all RFs at one station by either stretching or contracting them. We started this process for the $P s$ phase for the continental Mohorövičić discontinuity. Then, we repeated it progressively for all the deeper $P S$ phases analysed in this study (upper-lower oceanic crust interface and lower oceanic crust-mantle interface). Once the effect of the ray parameter discrepancy over the relative time of arrival of the Ps phases was removed, the azimuthal variations on the velocities could be measured with higher precision and they only reflected the anisotropic properties and the non-horizontality of the layers.

After removal of the azimuthal and ray parameter variations, we could determine the anisotropy parameters. We did this separately for the dipping layers and for the flat section. To characterize the anisotropic component in regions where the existence of dipping layers had been previously determined (southern $\sim 60 \mathrm{~km}$ of MASE array), we used a technique similar to the one used in most seismic refraction experiments. It consisted of directly measuring the azimuthal variation of the velocities of the waves to determine the fast azimuth direction $(\varphi)$, and cross-correlating the radial and the tangential components to define the magnitude of shear wave splitting $(\delta t)$ (Appendix A in the Supporting Information).

We recognized that some of the azimuthal dependence on the converted phase might be due to the dipping interfaces. We tested this by generating synthetic RFs in dipping and flat slab configurations to compare the isotropic and anisotropic effects on the signals. Fig. 3 shows synthetic RFs (Frederiksen \& Bostock 2000) for four cases (Table 1): (i) isotropic horizontal-layered media; (ii) anisotropic horizontal-layered media; (iii) isotropic dipping-layered media and (iv) anisotropic dipping-layered media. Radial and tangential RFs were complex for the anisotropic cases, and the $180^{\circ}$ periodicity is evident as expected from the discussion above.

In regions that can be approximated by horizontal layers, we averaged the results of a particle motion analysis and a waveform cross-correlation procedure (McNamara \& Owens 1993), except in the cases where the results of the two differed considerably. In such cases, the discrepancy is ascribed to difficulties in determining the initial Ps polarization direction, and thus the waveform crosscorrelation result alone was used. After these steps, the fast azimuth direction $(\varphi)$ and the time delay $(\delta t)$ of the $P s$ waves had been estimated for the dipping and horizontal sections.

Unlike most seismic anisotropy studies with RFs, which characterize the anisotropic component of a single relatively shallow layer, we seek to measure shear wave splitting in both the continental crust and the underlying oceanic slab. For this reason, it is essential to take into account that RFs carry the structural information from the conversion point of the $P S$ waves to the seismic station where these are observed (Fig. 4). Hence, a phase generated at a discontinuity at depth $h_{2}$ will convey the effects of the discontinuity at depth $h_{1}$. Similarly, a converted wave generated at a depth of $h_{3}$ will convey the effects of the discontinuities at depth $h_{2}$ and $h_{1}$ (assuming that $\left.h_{1}<h_{2}<h_{3}\right)$. Therefore, before measuring the shear wave anisotropy of a given layer, it is necessary to characterize and re-

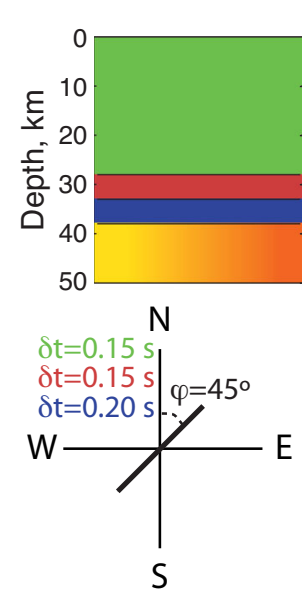

(a) Isotropic (b) Anisotropic
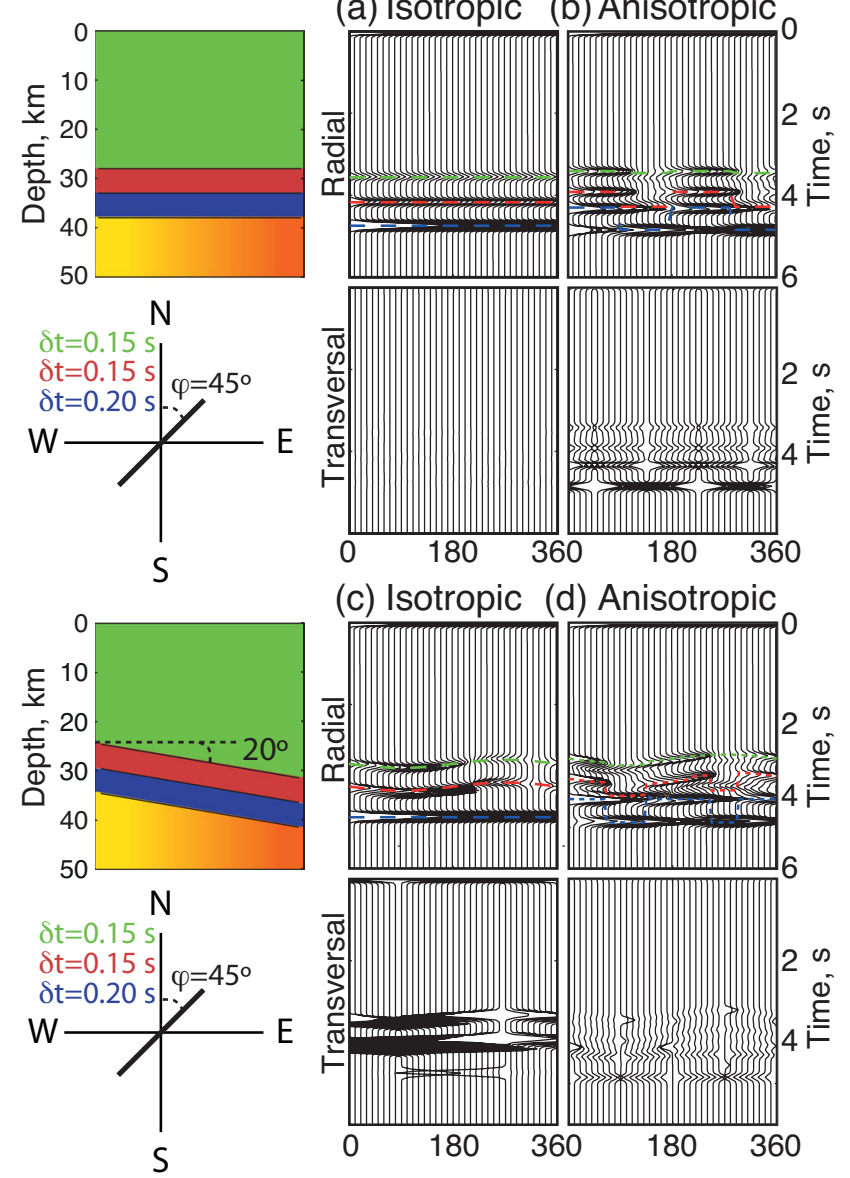

(c) Isotropic (d) Anisotropic

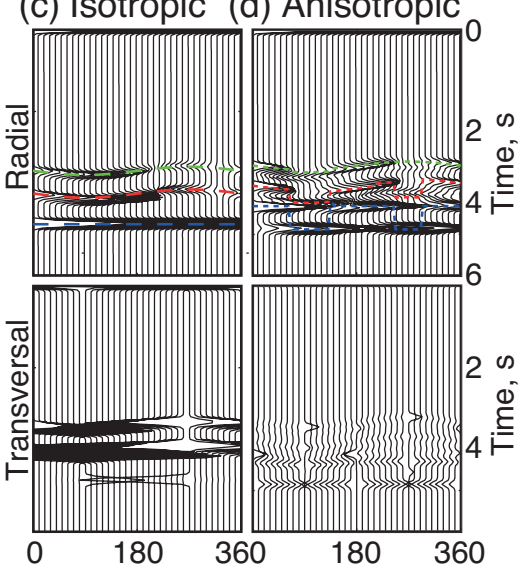

Figure 3. Synthetic RFs for (a) horizontal-layered isotropic media; (b) horizontal-layered anisotropic media; (c) dipping-layered isotropic media and (d) dipping-layered anisotropic media, dip $=45^{\circ}$ to the east. Properties of the layer models shown in the left are detailed in Table 1. For the anisotropic models, fast azimuth direction $\varphi=45^{\circ}, \delta t$ for each layer is colour-coded. Top panels, for each model, show the radial component, while the bottom, the transverse component. Dashed lines delineate the selected $P s$ phase for each layer discontinuity. Picking is done only in the radial component.

move the anisotropic effect of the preceding $P s$ phases by rotating the radial and tangential RFs to the fast and slow polarization directions of the overlying layers and by accounting for the shear wave splitting between the two orthogonal phases in question.

The $\varphi$ and $\delta t$ for each RF were assigned a quality ranking based on the signal-to-noise ratio and impulsiveness of the radial $P s$ phases. They were only considered useful when waveforms were similar in shape in the fast/slow coordinate system, when corrected particle motion was linearized and when the shear wave splitting time was within a coherent range. For this last quality control, we considered an upper time splitting limit of $0.35 \mathrm{~s}$ for the continental crust and $0.25 \mathrm{~s}$ for the upper and lower oceanic crusts, based on their average thickness and reasonable seismic velocities. Measurements of crustal anisotropy worldwide typically range between 0.1 and $0.3 \mathrm{~s}$ and average to $0.2 \mathrm{~s}$ (e.g. Kaneshima 1990; Silver \& Chan 1991; Silver 1996; Crampin \& Gao 2006). Furthermore, the crustal, upper limit set for the time delay in this study is consistent with earlier anisotropy measurements in this same region of flat slab subduction (Song \& Kim 2012a,b; Audet 2013; Huesca-Pérez et al. 2016). Final $\varphi$ and $\delta t$ values for a particular depth at each station were obtained by discarding low-quality estimates and averaging the remaining 
Table 1. Parameters for synthetic models shown in Fig. 3.

\begin{tabular}{|c|c|c|c|c|c|c|c|c|c|c|c|c|c|}
\hline \multirow[b]{2}{*}{ Thickness (m) } & \multirow[b]{2}{*}{$V_{p}\left(\mathrm{~m} \mathrm{~s}^{-1}\right)$} & \multirow[b]{2}{*}{$V_{s}\left(\mathrm{~m} \mathrm{~s}^{-1}\right)$} & \multirow[b]{2}{*}{$\rho\left(\mathrm{kg} \mathrm{m}^{-3}\right)$} & \multirow[b]{2}{*}{ Strike $\left(^{\circ}\right)$} & \multirow[b]{2}{*}{$\operatorname{Dip}\left(^{\circ}\right)$} & \multicolumn{2}{|c|}{ Model } & \multicolumn{2}{|c|}{ Estimated } & \multicolumn{2}{|c|}{ Model } & \multicolumn{2}{|c|}{ Estimated } \\
\hline & & & & & & $\varphi\left({ }^{\circ}\right)$ & $\delta t \quad(\mathrm{~s})$ & $\hat{\varphi}\left(^{\circ}\right)$ & $\hat{\delta} t \quad(\mathrm{~s})$ & $\varphi\left({ }^{\circ}\right)$ & $\delta t(\mathrm{~s})$ & $\hat{\varphi}\left({ }^{\circ}\right)$ & (s) \\
\hline Horizontal & & & & & & \multicolumn{4}{|c|}{ Isotropic } & \multicolumn{4}{|c|}{ Anisotropic } \\
\hline 28000 & 6000 & 3400 & 2700 & 0 & 0 & 0 & 0 & 0 & 0 & 45 & 0.15 & 12 & 0.10 \\
\hline 5000 & 6400 & 3700 & 2800 & 0 & 0 & 0 & 0 & 0 & 0 & 45 & 0.20 & 33 & 0.25 \\
\hline- & 8000 & 4600 & 3400 & 0 & 0 & 0 & 0 & 0 & 0 & 0 & 0 & 0 & 0 \\
\hline Dipping & & & & & & \multicolumn{4}{|c|}{ Isotropic } & \multicolumn{4}{|c|}{ Anisotropic } \\
\hline 5000 & 6400 & 3700 & 2800 & 0 & 20 & 0 & 0 & 0 & 0 & 45 & 0.20 & 39 & 0.26 \\
\hline- & 8000 & 4600 & 3400 & 0 & 0 & 0 & 0 & 0 & 0 & 0 & 0 & 0 & 0 \\
\hline
\end{tabular}

Notes: $V_{p}=P$-wave velocity, $V_{s}=S$-wave velocity, $\rho=$ density, $\varphi=$ fast azimuth direction, $\delta t=$ time delay, $\hat{\varphi}=$ estimated fast azimuth direction and $\hat{\delta} t=$ estimated time delay.

(a)

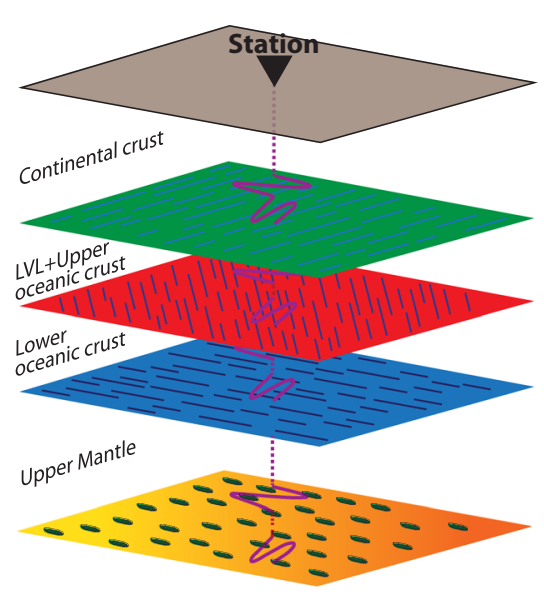

(b)

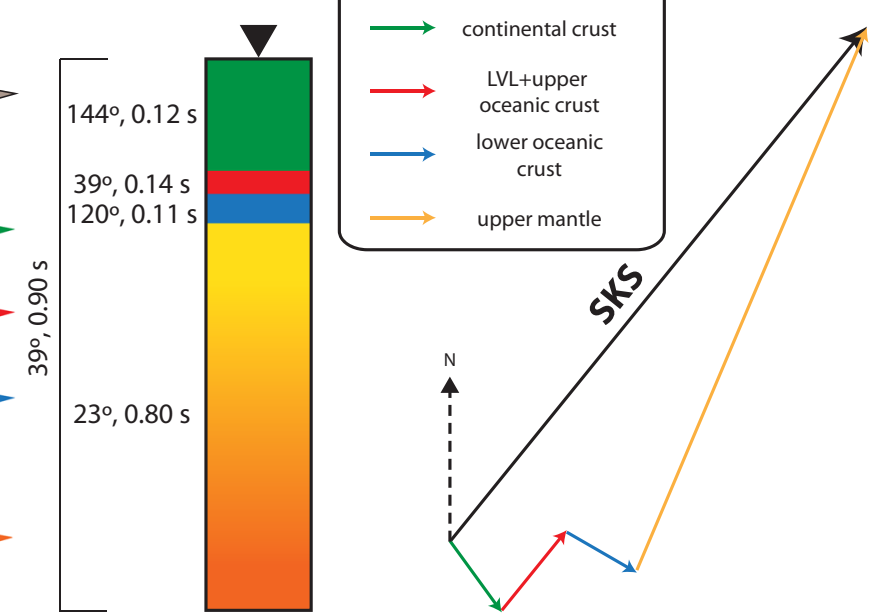

Figure 4. (a) Schematic anisotropy effect for each layer. (b) Illustration of the procedure used to constrain the upper-mantle anisotropy beneath station VEVI. Left: layer model for station VEVI, indicating the fast azimuth direction and the delay time. The total anisotropy parameters correspond to the SKS previously reported by Rojo Garibaldi (2011). Right: vector sum, each vector is colour-coded according to the layer (green = continental crust; red $=$ LVL+ upper oceanic crust; blue $=$ lower oceanic crust and yellow $=$ upper mantle). The vector direction corresponds to the fast azimuth direction, and the length, to the delay time. The sum of vector for all layers, including the unknown for the upper mantle, should be equal to the $S K S$ vector.

values over for the full backazimuth range. We used McNamara \& Owens' (1993) criterion as a reliability check of our final $\varphi$ and $\delta t$ estimates. This was done by stacking all available high-quality RFs in the fast/slow coordinate system as defined by the fast polarization direction of the recently characterized layer and station. All selected RFs were multiplied by +1 or -1 depending on their polarity to ensure that every trace presented a positive polarity of the $P s$ phase of interest in both the fast and slow components of motion. In most cases, RFs showed an increase in their waveform similarity and a well-defined phase splitting, which suggested an appropriate $\varphi$ and $\delta t$ estimation.

Once the continental and oceanic crust anisotropy were established, their influence was removed from the previous SKS measurements made by Rojo Garibaldi (2011) along the same array of seismic stations. The $S K S$ measurement is the cumulative total of the anisotropy all along the upgoing $S$-wave path through the mantle, the slab (where present) and the continental crust (e.g. Silver \& Chan 1991; Silver 1996; Savage 1999; Long \& Silver 2008). The removal process was done by representing the $S K S$ anisotropy as a vector, with its direction given by $\varphi$ and a length equal to $\delta t$. This vector represented the anisotropy for the crust and the upper mantle. In the southern segment of the MASE array, it also included the anisotropy for the slab. We then subtracted the anisotropy vectors of the other corresponding layers from the $S K S$ anisotropy vector to provide us with an approximate measure of the seismic anisotropy contained exclusively in the upper mantle. Fig. 4 shows the schematic process of such a removal for one station. The uncertainties $\left(\sigma_{\varphi}, \sigma_{\delta t}\right)$ of the $S K S$ splitting measurements $\left(\sigma_{\delta t} \approx 0.2 \mathrm{~s}\right)$ include, and are actually larger than, the uncertainties measured using RFs in the continental and oceanic crust $\left(\sigma_{\delta t} \leq 0.1 \mathrm{~s}\right)$. In this study, the splitting parameters $(\varphi, \delta t)$ reported for the upper mantle in Table B4 in the Supporting Information are obtained by subtracting the anisotropy of the crust (continental and oceanic where present as given in Tables B1-B3 in the Supporting Information) determined from RF from the $S K S$ measurements. The uncertainties $\left(\sigma_{\varphi}, \sigma_{\delta t}\right)$ reported in Table B4 in the Supporting Information, however, are the uncertainties from the original $S K S$ study and are considered to represent the uppermost bound.

We followed the described procedure for the four models shown in Fig. 3 with parameters listed in Table 1. No anisotropy was detected for the isotropic horizontal-layered model, while for the anisotropic case, values for $\varphi$ and $\delta t$ were roughly recovered. A $\varphi$ was detected for the isotropic case with dipping layers. However, $\delta t$ was equal to zero for all layers, with isotropy as expected. 
$\varphi$ estimates were not accurately recovered for the anisotropic dipping layers. This suggested that some effect from the nonhorizontality of the layers affects its estimation, but the form of $\delta t$ was recovered if not the exact values. Therefore, for our analysis we only considered $\delta t$ estimated from RFs in which the waveforms of the fast and slow components are similar, in order to reduce the effect introduced by the dipping of the layers.

\section{RESULTS AND DISCUSSION}

The obtained anisotropy parameters are listed along with their corresponding standard errors in Appendix B in the Supporting Information. Shear wave splitting measurements may have been contaminated by the scattering caused by small-scale heterogeneities in the lithosphere, which under certain circumstances may have induced a 'false' splitting in our data that was not necessarily correlated between events (Langston 1989; Randall \& Owens 1994). To limit these effects, we described the relationship between our observations and their spatial variation by a loess smoothing function. This function was based on a classic least-squares inversion and attempted to find a general description of the data by a global assumption of the shape. In this case, a quadratic and its locally weighted mean were found to best fit the data (e.g. Efron \& Tibshirani 1993). With this method, we were able to provide local descriptions of the average anisotropic properties of the medium and identify patterns in its variations. Gilje \& Thomsen (1970) homogenization criterion was then used to laterally classify our observations based on their fast direction variance.

\subsection{Continental crust}

There are several mechanisms for generating hexagonal anisotropy in the continental crust, including alternating layers with contrasting elastic properties, aligned minerals in the proximity of crustal shear zones or metamorphic terranes (Balfour et al. 2005; Boness \& Zoback 2006) and stress-aligned cracks in sedimentary rocks (Crampin 1990; Hudson 1994; Crampin \& Zatsepin 1997; Crampin \& Chastin 2003; Boness \& Zoback 2004). In the case of aligned cracks, the fast direction is associated with the crack orientation whereas the time delay is related to the density and consistency of cracks. Alternately, if anisotropy is the outcome of shear fabric, the anisotropic component depends on the direction of fabric and degree of shearing (Cochran et al. 2006).

This anisotropic component of the continental crust was characterized in a total of 71 stations out of the 100 that composed the MASE array due to the limited amount of RFs available for processing, and the difficulty to identify impulsive $P s$ phases in various traces. In region A of the continental crust (Fig. 5), the estimated fast azimuth displays a consistent NE-SW orientation. However, the fast azimuth vectors exhibit a slight rotation toward to the E-W direction near the border limit of the Xolapa terrane, the GuerreroMorelos platform and the Mixteco terrane. The NE-SW orientation displayed by the first part of the array is consistent with the regional stress field, which is expected to have a maximum compression axis in this direction as a consequence of the subduction process (Suter 1991). This anisotropy is most likely the result of aligned microcracks, a process referred to as extensive dilatancy anisotropy (Crampin 1994). In this simplified model, it is possible to approximate such a setting as transverse isotropy (TI) with a horizontal symmetry axis in which pre-existing cracks that are approximately parallel to the direction of maximum compressive horizontal stress may have been opened while others are closed. We suggest that the anisotropy direction near the boundary of the Xolapa terrane is influenced by the E-W to WNW-ESE striking shear zone that bounds it (Solari et al. 2007).

Further into the array, within zones $\mathrm{B}$ and $\mathrm{C}$ of the continental crust (Fig. 5), we identifiy a subregion exhibiting a relatively highanisotropy percentage $(\sim 1.5$ per cent $)$ confined to $105-140 \mathrm{~km}$ from the coast (Fig. 6d). Previous poroelastic modeling of slow-slip events (SSEs) observed in southern Mexico (Villafuerte 2014) has revealed that both the 2006 and 2010 SSEs induced a confining pressure reduction in this region that could be functioning as a dilatancy mechanism (Fig. 6e). This strain configuration could be translated as a proclivity of the continental crust to fracture and stimulate the existence of open cracks, which may in turn enhance the shear wave splitting in this part of the array and explain the observed contrasts in anisotropy percentages. However, the increased dilatancy modeled by Villafuerte (2014) was not solely limited to the region where we find an elevated anisotropy percentage, so there must be additional, undetermined, mechanisms that contribute to the shear wave splitting observed in this part of the array.

Within the Trans-Mexican Volcanic Belt (TMVB, zones D-I; Fig. 5), anisotropy is most likely associated with open cracks, volcanic vent alignment and the dominant trend of faulting. The main tendency of the fast azimuth directions inside this region display a parallelism with the general direction of the Neogene extensional faulting, which has an ENE-WSW and E-W preferred orientation (Ferrari et al. 2012). Also, a comparison with numerous other studies along the MASE array suggests that the existence of fluids and partial melts generated by the subduction process play an important role in the increased observed shear wave splitting (Fig. 6). Zones of high conductivity (Jödicke et al. 2006), low velocity (Iglesias et al. 2010) and high attenuation (Chen \& Clayton 2009) are consistent with the anomalously high-anisotropy percentage zone found in the crust below the TMVB. This last correlation leads us to believe that the vertical fracturing produced by the ascent of lower density materials is an important mechanism that contributes to the observed high shear wave time splitting within this volcanic province.

\subsection{Oceanic crust}

Reliable measurements for the LVL and upper and lower oceanic crusts were obtained in 34 MASE stations (Fig. 7). They were considered reliable if the subducting plate was observed in the RF signal and the continental crust anisotropy was successfully established. Fast azimuth direction estimates for the LVL and the upper oceanic crust display a more complex configuration than that observed for the continental crust, exhibiting significant variability and contrasts at smaller distances. Our interpretation of these inconsistencies is that the high heterogeneity and structural complexity of this layer is a direct consequence of the fragility of the low strength hydrous minerals that have been proposed to compose it (e.g. talc; Kim et al. 2010), which, under a significant amount of stress, could result in a highly heterogeneous fracturing pattern. The lower oceanic crust, on the other hand, appears to be less heterogeneous, as previously suggested by Kim et al. (2010). Given the existing high confining pressure at this depth, most of the anisotropy within the lower oceanic crust is likely associated with the presence of foliated serpentine minerals and with the preferred orientation of olivine and pyroxene crystals generated during the formation and spreading of the oceanic plate. These mechanisms would explain the increased consistency in the estimated fast azimuth directions. This is because 

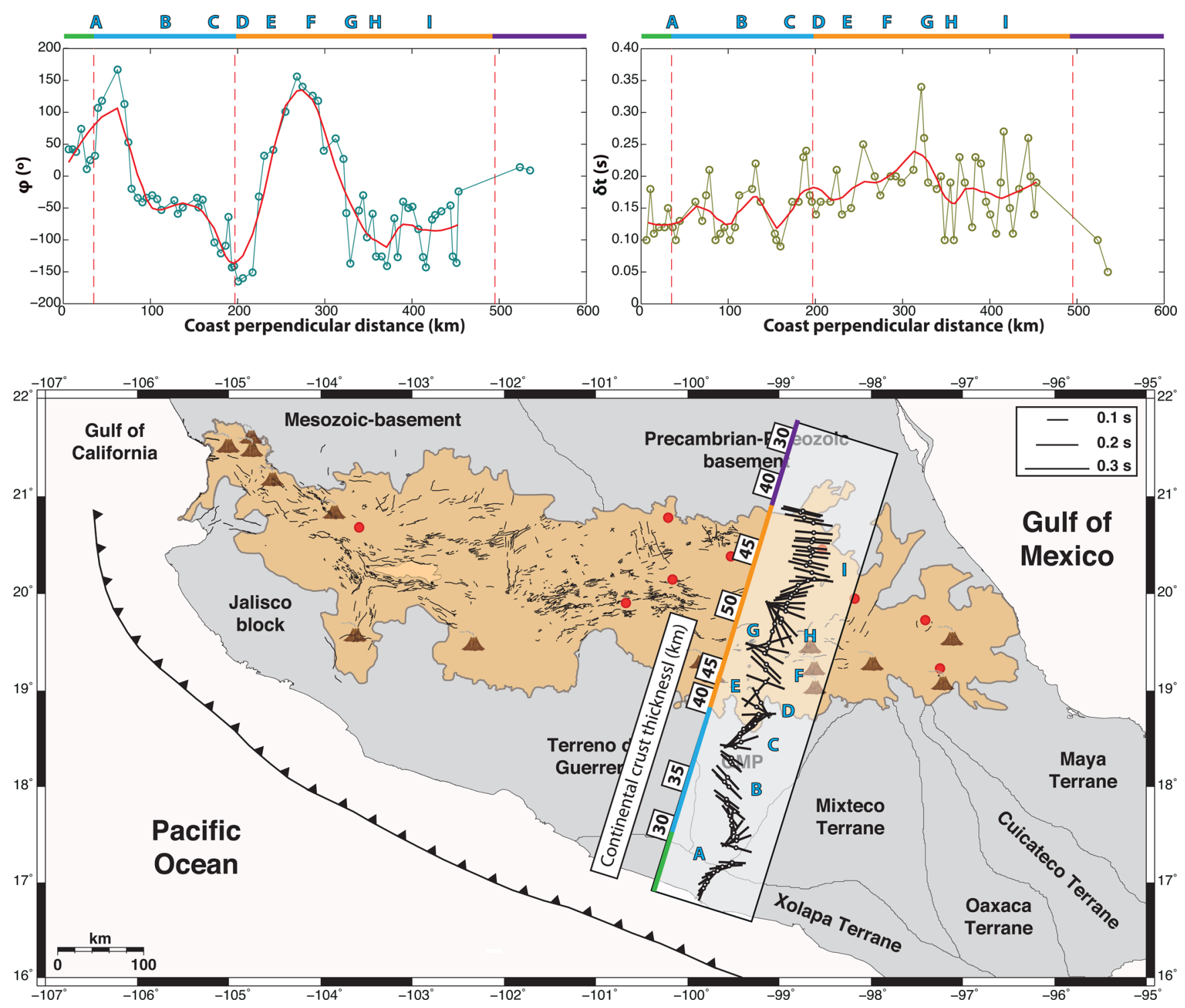

Figure 5. Variation of the anisotropy parameters $\varphi$ and $\delta t$ for the continental crust. The thick red lines represent the loess smoothed descriptions of the parameters. The coloured bars at the top of the panels indicate the tectonostratigraphic terranes (green: Xolapa terrane; blue: Guerrero-Morelos platform; yellow: TMVB and purple: Precambrian-Palaeozoic basement). The map shows the smoothed anisotropy measurements projected onto a horizontal plane. The size of the bars represents the magnitude of the observed shear wave splitting, whereas their orientation indicates the fast polarization direction. The letters on the map mark changes in the fast polarization direction, identified using Gilje \& Thomsen's (1970) homogenization criterion. Also shown on the map are the main crustal units and the most prominent tectonic and volcanic structures of the Trans-Mexican Volcanic Belt (the black thin lines indicate mapped faults and the red dots indicate the main calderas; Ferrari et al. 2012). Brown triangles show location of active volcanoes.

once a mineral preferred orientation fabric is formed, it is highly unlikely to be destroyed, unlike in the case of fracturing. The resultant anisotropy is preserved during the subduction process to at least a depth of $400 \mathrm{~km}$, where the pressure and temperature $(P-T)$ condition promote the transformation of olivine to wadsleyite (Heiffrich \& Wood 2001).

Detailed mapping of inverted seismic parameters along the MASE array has previously revealed high Poisson's and $V_{p} / V_{s}$ ratios (2.3-2.8) in the subhorizontal region of the upper oceanic crust on top of an unaltered lower oceanic crust (Kim et al. 2010). In most cases, high Poisson's and $V_{p} / V_{s}$ ratios have been interpreted as regions with a high fluid content or high pore pressure (Kodaira et al. 2004; Audet et al. 2009; Peacock et al. 2011). An alternate and complementary hypothesis is that the high $V_{p} / V_{s}$ ratio due to a low $S$-wave velocity can be related to a preferred mineral alignment in the medium, a process expected to be particularly marked in the case of serpentine (Mainprice \& Ildefonse 2009; Bezacier et al. 2010). To analyse this relationship further, Fig. 8 (upper) shows a comparison of $V_{p} / V_{s}$ ratios (Kim et al. 2010) and the estimated anisotropy percentages for the upper and lower oceanic crusts of the first $\sim 160 \mathrm{~km}$ of the MASE array. Assuming the existence of a single $V_{p} / V_{s}$ value below each station may be an oversimplification, since in an anisotropic medium there are at least three wave velocities $\left(V_{p}\right.$, $V_{s 1}$ and $\left.V_{s 2}\right)$, and consequently two $V_{p} / V_{s}$ ratios $\left(V_{p} / V_{s 1}\right.$ and $\left.V_{p} / V_{s 2}\right)$ that vary with direction (e.g. Mavko et al. 1998). Although it is not possible to separate the two, $V_{p} / V_{s}$ gives the average of the two.

High-anisotropy percentages found in the LVL and the upper and lower oceanic crusts exist in zones with low $V_{p} / V_{s}$ values, and thus high $S$-wave velocity (grey dashed boxes Fig. 8). Two simple TI models are able to explain this observation, one with a horizontal 
(a)

Resistivity in $\Omega \mathrm{m}$

$\begin{array}{llllllllll}1000 & 500 & 200 & 100 & 50 & 20 & 10 & 5 & 2 & 1\end{array}$

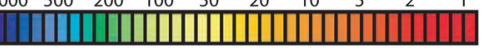

TMVB

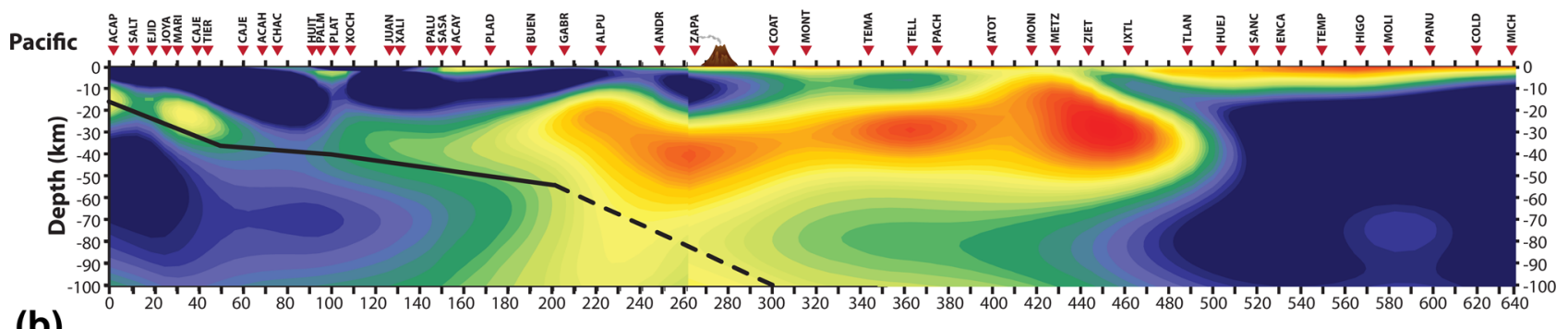

(b)

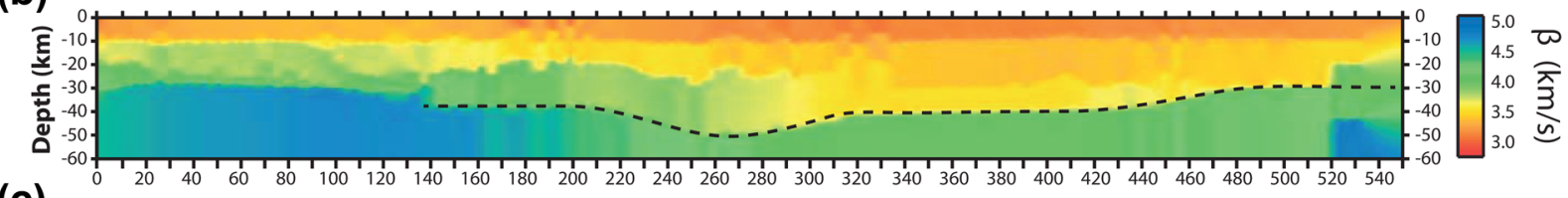

(c)
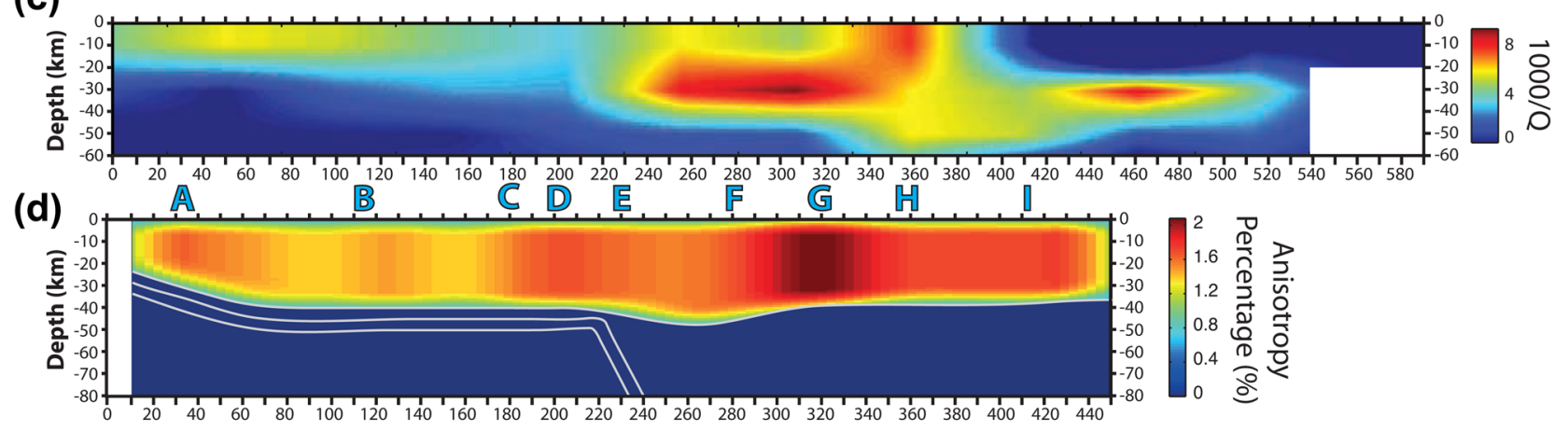

(e)

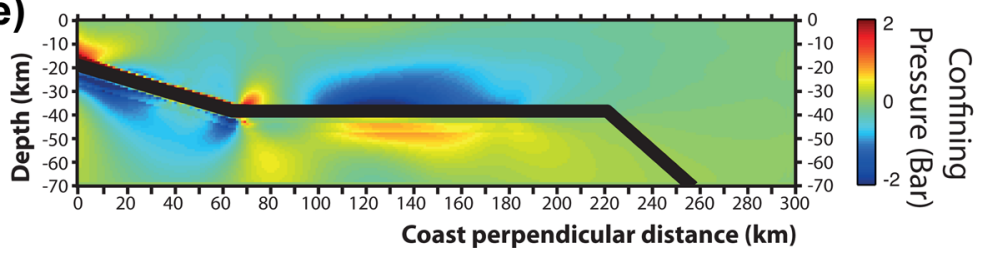

Figure 6. Comparison of the geophysical structures along the MASE array, obtained in a range of studies. (a) Resistivity measurements from a magnetotelluric survey (Jödicke et al. 2006). (b) Shear wave velocity along the MASE array from a surface wave study (Iglesias et al. 2010). (c) Attenuation inversion results from $P$ waves (Chen \& Clayton 2009). (d) Anisotropy percentages observed in this study. (e) Confining pressure, $P_{\mathrm{c}}$, distribution induced by the $2006 \mathrm{SSE}$ (Villafuerte 2014).

symmetry axis and the other one with a vertical symmetry axis (Fig. 8, bottom). Our RFs are derived from teleseismic events, so the rays analysed in these time-series traveled vertically through the structure. The observed shear wave splitting is therefore much more sensitive to a configuration with a horizontal symmetry axis than to one with vertical symmetry axis. The average shear wave velocity in a TI medium with horizontal symmetry axis, where a 'fast' and a 'slow' shear wave exists, is larger than the shear wave velocity in a TI medium with vertical symmetry axis, where only 'slow' $S$ waves exist. This explains the regions in which we observe a low $V_{p} / V_{s}$ value and, at the same time, a high-anisotropy percentage (vertical symmetry axis) and vice versa (horizontal symmetry axis). The $V_{p} / V_{s}$ ratios shown in Fig. 8 were obtained by Kim et al. (2010), assuming fixed $P$-wave velocities, making its variation due to anisotropy irrelevant. These observations were in agreement with those of Wang et al. (2012), who analysed the $V_{p} / V_{s}$ and anisotropy relationship in fractured media for a wide range of confining pressures.

The same correlation is observed by analysing the $V_{p} / V_{s}$ ratios computed for the continental crust along the MASE array (Fig. 9).
A region with a low $V_{p} / V_{s}$ ratio in the tomography (Huesca-Pérez \& Husker 2012) is coincident with a region of the continental crust that exhibits a low average anisotropy percentage in this study. This may seem contrary to the observed relationship with the results of Kim et al. (2010), in which low $V_{p} / V_{s}$ ratios corresponded to highanisotropy percentages. However, their tomography was mostly limited to horizontal ray paths, for which the high shear wave velocity (low $V_{p} / V_{s}$ ratio) might also be a consequence of waves propagating parallel to the foliation of the medium, while it would be expressed as a low shear wave time splitting for the vertical paths in this study.

Based on the previous analysis, we argue that anisotropy may have an influence in $V_{p} / V_{s}$ ratios and that its consideration may be relevant when studying the rheology of the medium. It is important to clarify however, that this observation does not undermine the role of fluids or pore pressure in $V_{p} / V_{s}$ ratio estimations. The anomalously high $V_{p} / V_{s}$ ratios, as the ones observed by Kim et al. (2010), and the anisotropy observed throughout the medium could only be found by a combination of slow polarized shear waves and the presence of water and hydrous minerals or high pore pressure. This suggests that there is high pore pressure that maintains cracks open, creating 


\section{Upper oceanic crust Lower oceanic crust}
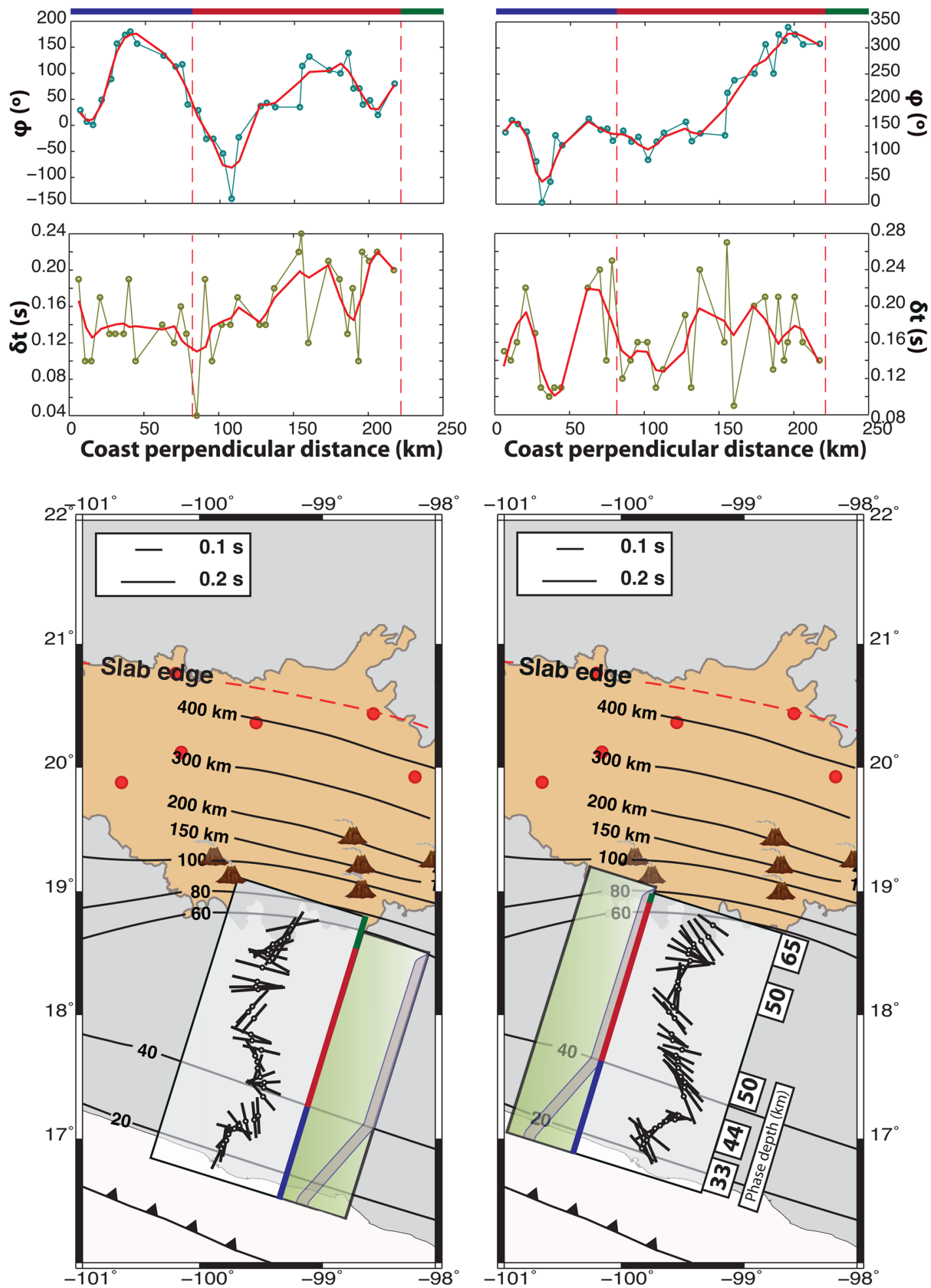

Figure 7. Variation of the anisotropy parameters $(\varphi$ and $\delta t)$ for the LVL + upper and lower oceanic crusts. The thick red lines represent the loess-smoothed descriptions of the parameters and the coloured bars on the top of the panels indicate the changes in the dip angle of the slab (blue is where the plate subducts at an angle of $19^{\circ}$, red is the subhorizontal region of the slab and green is where the oceanic plate plunges steeply into the mantle). The maps show the smoothed measurements projected into a horizontal plane. The size of the bars represents the magnitude of the observed shear wave splitting, whereas the orientation indicates the fast polarization direction. A schematic model of the horizontal slab geometry is shown alongside the obtained measurements. 


\section{(a) Upper oceanic crust}

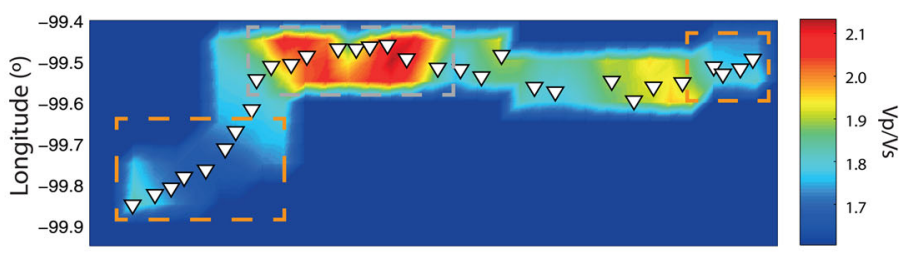

\section{(b) Lower oceanic crust}
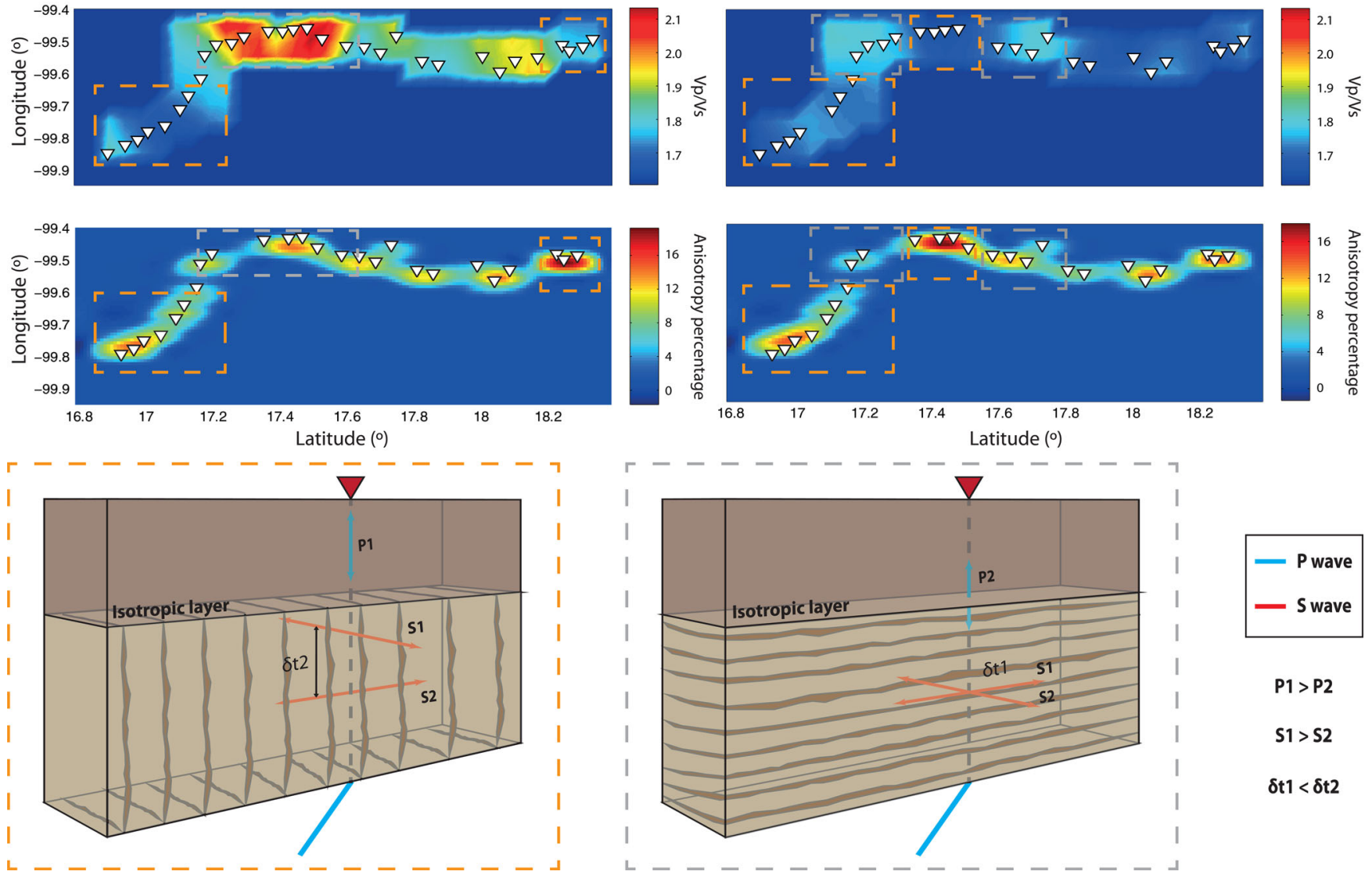

Figure 8. Top: $V_{p} / V_{s}$ ratio (Kim et al. 2010). Middle: anisotropy percentage variations within the LVL+ upper and lower oceanic crusts from this study. The subhorizontal part of the oceanic crust starts at latitude $17.2^{\circ}$. The orange dashed boxes indicate the regions that exhibit the highest anisotropy percentages, whereas the grey dashed boxes indicate the regions where the estimates of $V_{p} / V_{s}$ ratios are highest. Each anisotropy percentage variation plot is generated using the spline interpolation scheme. Bottom: schematic models of a TI medium that can explain the patterns of the seismic parameters: orange dashed box-horizontal symmetry axis and grey dashed box-vertical symmetry axis. For a wave propagating vertically, both the average $P$ - and $S$-wave velocities are greater in the medium with a horizontal symmetry axis than in the one with a vertical symmetry axis. The shear wave splitting is also larger in the medium with a horizontal symmetry axis.
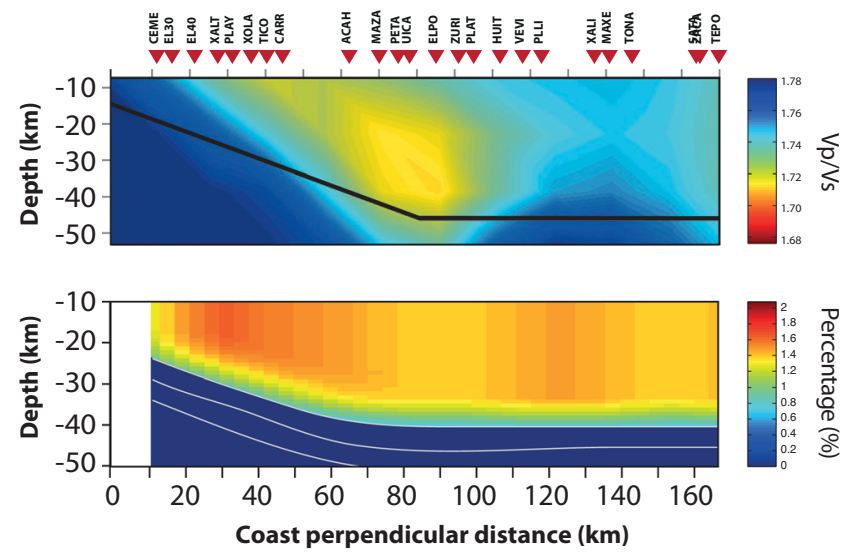

Figure 9. (Top) $V_{p} / V_{s}$ ratio (Huesca-Pérez \& Husker 2012) compared with (bottom) anisotropy percentage variations within the continental crust along the first $170 \mathrm{~km}$ of the MASE array. a high $V_{p} / V_{s}$ ratio and perpendicular permeability anisotropy, with the first effect enhancing the second one.

\subsection{Upper mantle}

At high confining pressures and high temperatures, where no microfractures and open cracks can exist, anisotropy is controlled by the preferred orientation of (olivine) minerals. Plastic and viscous flow is the foremost mechanism that systematically orients crystals in the upper mantle, and is responsible for the anisotropy of its aggregates (Mainprice \& Nicolas 1989). Early interpretations of shear wave splitting with $S K S$ waves were made under the assumption that the fast polarization direction is parallel to the asthenospheric flow (e.g. Christensen 1984; Nicolas \& Christensen 1987; Zhang \& Karato 1995). Such premises came from laboratory experiments that involved dry olivine samples. However, subsequent work found that by using wet olivine, the relationship between the orientation of fast polarized shear waves and the flow direction changed, such that the fast axis aligns perpendicular to the flow (Jung \& Karato 2001). This configuration was known as olivine type-B, whereas the relationship of dry olivine is type-A. Typically, type-A olivine 
(a)

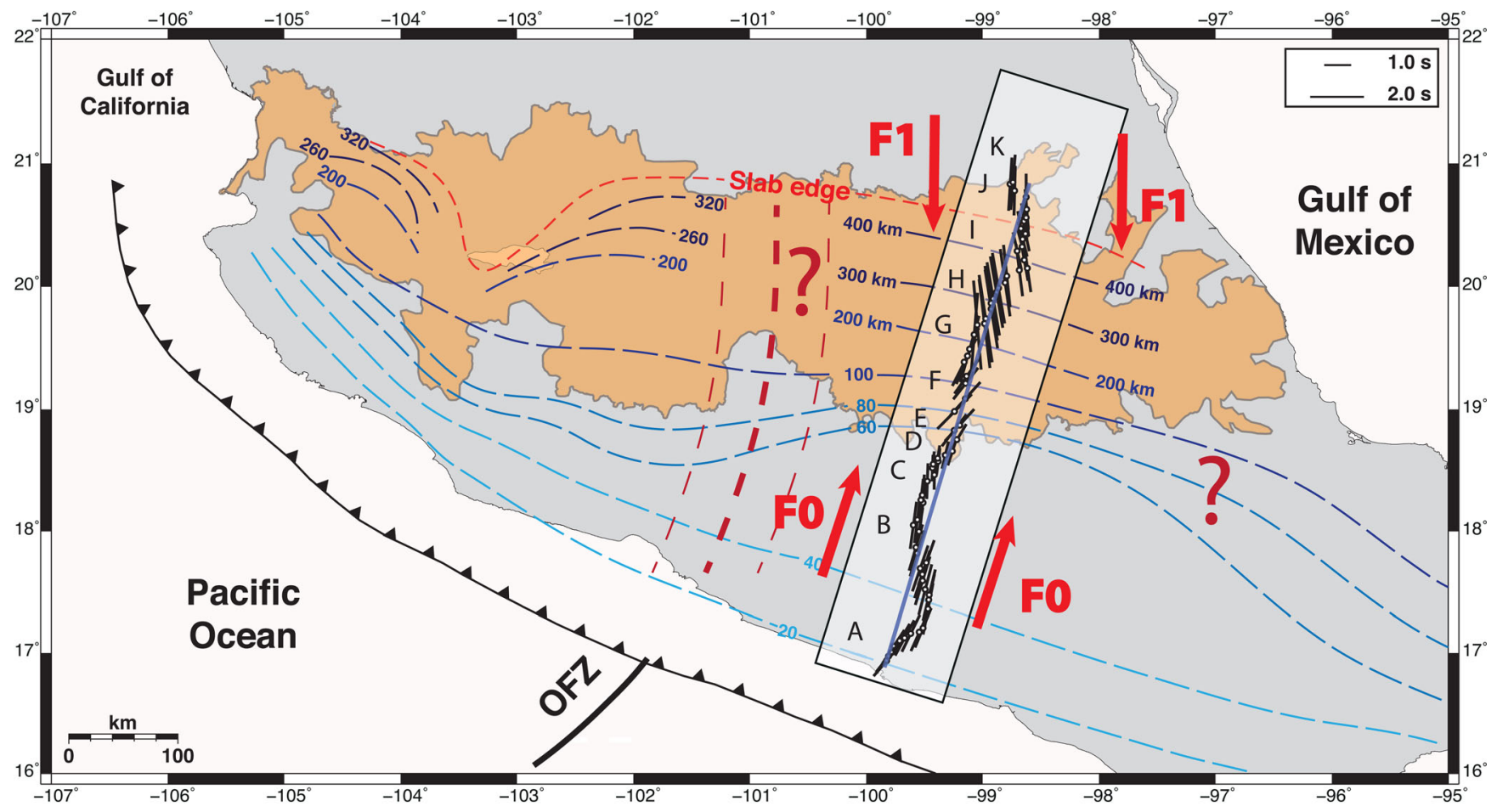

(b)

TMVB

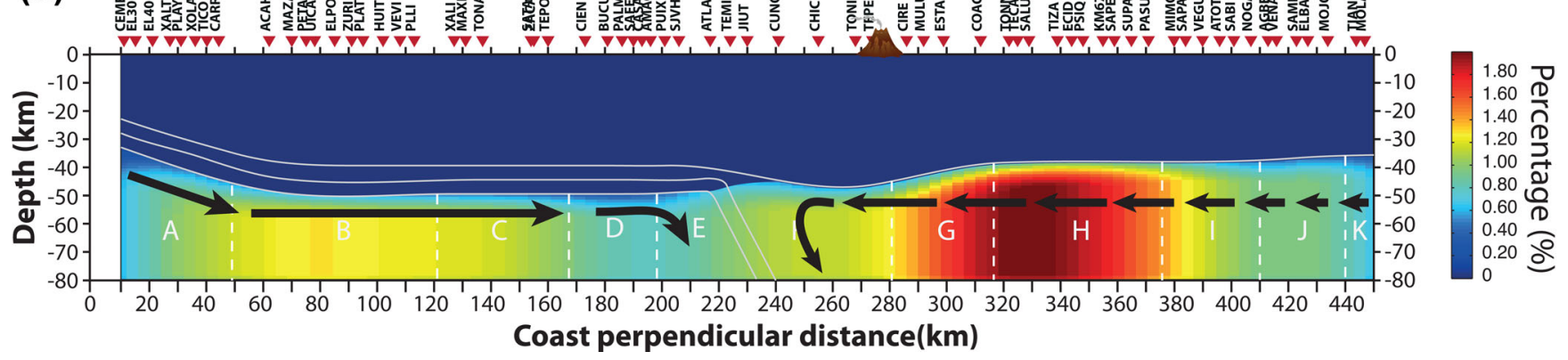

Figure 10. (a) Map showing the smoothed anisotropy measurements for the upper mantle projected into a horizontal plane. The length of the bars in the map represents the magnitude of the observed delay times, whereas the orientation indicates the fast polarization directions. The extension of the Orozco Fracture Zone (OFZ) projected beneath the North American plate is shown as the dashed red line along with two thinner lines indicating the approximate width of the possible fracture zone (Blatter \& Hammersley 2010). The eastern region where the existence of another tear in the plate has been suggested, but not confirmed, is pointed out with a question mark. The red arrows within the map indicate the different asthenospheric flow directions suggested by this study. F0 represents the 2-D, subslab entrained flow induced by the drag of the subducting plate, and F1, in the northern part of the array, represents corner flow within the mantle wedge driven by the subduction process. The blue line across the measurements denotes the section presented in the lower part of the image. (b) Depth profile showing the estimated anisotropy percentages along the MASE array. Larger delay times in the southern part of the array $(\sim 1.2$ per cent) are observed in the upper mantle under the flat slab, where mantle flow is likely to be completely horizontal. Smaller delay times $(\sim 0.9$ per cent and 0.7 per cent $)$ are observed where the slab is dipping, and consequently the asthenospheric flow has both a horizontal and a vertical component. The black arrows represent subslab entrained flow under the southern half of the array, and corner flow within the mantle wedge beneath the northern half of the array.

is found in regions of low water content, relatively small amounts of stresses and high temperatures, while type-B olivine is characteristic of enriched water settings, high stresses and low temperature (Jung \& Karato 2001; Karato et al. 2008; Long 2009a). In subduction zones, the mantle wedge tip frequently meets the conditions required for the existence of type-B olivine, whereas type-A (or similar) is found throughout the mantle wedge core (Kneller et al. 2005). However, the observed anisotropy in the upper mantle beneath the MASE array may be due to type-A olivine, because of the young age $(\sim 14 \mathrm{Ma})$ of the Cocos plate and its high temperature
(Pardo \& Suárez 1995; Manea et al. 2005, 2006; Husker \& Davis 2009; Rojo Garibaldi 2011).

Fig. 10 shows the anisotropy for the upper mantle. In the southern part of the array, before the oceanic plate plunges steeply into the mantle, $\varphi$ values are oriented NE-SW and are consistently perpendicular to the trench, while $\delta t$ estimates display a slight variability depending on the distance from the coast. We propose that the orientation of the fast axes is a result of subslab entrained mantle flow. This interpretation is consistent with earlier work using data from the MASE array (Rojo Garibaldi 2011; Bernal-López et al. 2016) 
and from the Mexican permanent network (Ponce Cortés 2012; van Benthem et al. 2013). Within this region, we are able to distinguish two areas with a relatively weak anisotropy percentage (zones $\mathrm{A}$ and D-E in Fig. 10b). We suggest that this might be a consequence of the relationship between the tilt of the flow and the nearly vertical incidence of the $S K S$ waves used to measure the original shear wave splitting parameters. Given that the slab drags the mantle underneath, slab geometry controls both the direction and the inclination of the flow. Below the flat slab region, mantle flow only has a horizontal component. Where the slab is dipping, however, mantle flow has both horizontal and vertical components. As the dip of the slab increases, the horizontal component of flow decreases and so does the measurable delay time considering that the shear wave splitting method can only resolve the horizontal component of the flow (Fig. 10b). SKS shear wave splitting measurements are usually interpreted to result from azimuthal anisotropy in the upper mantle under the station (e.g. Long 2009b) and this is the view we adopted in this study. Alternatively, if a medium with TI and hexagonal symmetry is assumed, and if the $S K S$ path is subparallel to the dipping symmetry axis, a small splitting time may result.

Further north, where the oceanic slab is no longer visible in the RFs, a slight variation in the behaviour of the fast azimuth directions for the upper mantle is evident given that these become oriented N-S. Long \& Silver (2008) suggested that mantle flow in the wedge is controlled by the competition of two flow fields: (1) a 2-D corner flow that depends on the viscosity between the subducting slab and the mantle around it (poloidal flow); and (2) a 3-D return flow around the slab edge that is induced by migration of the trench (toroidal flow). In the area of our study, the fast azimuthal direction and the amount of splitting (Fig. 10), suggest the predominance of corner flow induced by downdip motion of the slab over a 3-D return flow. This interpretation is consistent with Bernal-López et al. (2016). They proposed that the different orientation of the fast axes between the southern and northern ends of the MASE deployment can be explained because the TMVB is not subparallel to the MAT (Gil 1981; Suárez \& Singh 1986; Ferrari et al. 2012). It is important to take into account that due to the low resolution achieved by RFs in extremely dipping layers, it was not possible to quantify and remove the anisotropy contribution of the subducting plate beneath the mantle wedge. Therefore, the results for this region might have been partly contaminated by the oceanic plate anisotropy.

The anisotropy percentages in the northern half of the MASE array show some differences in strength (Fig. 10b). Zone F shows a small anisotropy percentage. In the region immediately to the north (zones $\mathrm{G}$ and $\mathrm{H}$ in Fig. 10b), the observed anisotropy percentages went up to $\sim 2$ per cent. Finally, the anisotropy percentages drop down to $\sim 0.6$ per cent at a distance of $380 \mathrm{~km}$ from the coast (zones I, J and K in Fig. 10b). The strong anisotropy percentages observed in zones $\mathrm{G}$ and $\mathrm{H}$ may possibly occur in the region where corner flow is most coherent, as indicated by the consistent orientations of the fast axes in Fig. 10(a).

It has been suggested that a slab-tear exists along the eastern projection of the Orozco Fracture Zone as has been previously suggested (DeMets \& Wilson 1997; Dougherty et al. 2012; Stubailo et al. 2012). If this is the case, then slab rollback (Gómez-Tuena et al. 2007) should induce toroidal flow through such a tear. Our anisotropy data do not provide any evidence for flow through the tear, but this is most likely a consequence of the fact that the MASE array was deployed too far east of the proposed tear. As it happens, seismic coverage of the area around the proposed tear is sparse (van Benthem et al. 2013). The only existing station near the proposed tear is ZIIG from the Mexican permanent network.
The fast axis at that is oriented NNW-SSE (van Benthem et al. 2013; Valenzuela \& León Soto 2017) and is clearly different from the trench-perpendicular fast axes observed at stations located to the east (this study; Rojo Garibaldi 2011; Ponce Cortés 2012; van Benthem et al. 2013; Bernal-López et al. 2016; Valenzuela \& León Soto 2017), over the flat slab. Data from new stations are needed, however, in order to establish that the anisotropy pattern at ZIIG is caused by flow through a slab tear. For comparison, it should be noted that León Soto et al. (2009) documented toroidal flow around the western edge of the Rivera slab and also flow through the tear between the Rivera and Cocos slabs, but these observations were only available at a handful of stations. Otherwise, anisotropy at stations located directly over the Rivera slab is consistent with both subslab entrained flow and with corner flow within the mantle wedge (León Soto et al. 2009).

\section{CONCLUSIONS}

Vertically averaged shear wave anisotropy was quantified using $P$ to $S$ converted phases generated at different depths along a 100 station broad-band array across southern and central Mexico using a direct characterization technique, a particle motion analysis and a cross-correlation procedure. Our results were then used to remove the contribution of the crustal anisotropy from a previous $S K S$-wave splitting study along the same array of seismic stations.

Anisotropy measurements for the continental crust define a complex pattern, suggesting that multiple factors on numerous scales control the stress field in the crust in central and southern Mexico. Within the range of observations for this layer, we were able to distinguish four major ones: (1) fast azimuthal orientations quasiparallel with the convergence direction of the Cocos and North America plates, for the first $80 \mathrm{~km}$ of the array, (2) two subregions with relative low-anisotropy percentage ( $\sim 1.3$ per cent) confined to 80-105 and 140-170 km from the coast, (3) a dominant parallelism between the fast polarization directions and the ENE-WSW and E-W trending of the extensional faults within the TMVB and (4) high-anisotropy percentages in central Mexico ( $\sim 2$ per cent) which we attribute to the vertical fracturing of the crust caused by fluid ascent and partial melts (Jödicke et al. 2006; Chen \& Clayton 2009; Iglesias et al. 2010).

Moving from the continental crust to the subducted oceanic crust, we found a relationship between $V_{p} / V_{s}$ ratios and anisotropy. High shear wave splitting values in the upper and lower oceanic crusts correspond to zones where previous studies determined high $S$-wave velocities. We explained this observation with a TI model with a horizontal symmetry axis in which the existence of fast-polarized shear waves may result in an overestimation of the average shear wave velocity and reveal itself as a low $V_{p} / V_{s}$ ratio and high-anisotropy percentage. The same correlation was observed between estimated continental crust anisotropy and the velocities in a previous tomographic study (Huesca-Pérez \& Husker 2012).

The anisotropy in the upper mantle appears to be controlled by type-A olivine preferred orientations. In the southern part of MASE, before the plate dips steeply into the mantle, the observed fast azimuthal directions suggest the existence of subslab entrained flow perpendicular to the trench, induced by the drag of the plate. Corner flow within the mantle wedge is consistent with the anisotropy pattern observed in the northern part of the array. The change in the orientation of the fast axes between the southern (NE-SW axes) and northern ( $\mathrm{N}-\mathrm{S}$ axes) ends of the array is explained because the TMVB is not subparallel to the MAT. 


\section{ACKNOWLEDGEMENTS}

We thank the editor Saskia Goes and an anonymous reviewer whose comments greatly improved this manuscript. We thank the MASE team that installed and maintained MASE stations. Funding was possible through projects Conacyt 177676 and UNAM-PAPIIT IN110913. The first author had a scholarship from Conacyt.

\section{REFERENCES}

Ammon, C.J., 1991. The isolation of receiver effects from teleseismic P waveforms, Bull. seism. Soc. Am., 81, 2504-2510.

Audet, P., 2013. Seismic anisotropy of subducting oceanic uppermost mantle from fossil spreading, Geophys. Res. Lett., 40, 173-177.

Audet, P., Bostock, M.G., Christensen, N.I. \& Peacock, S.M., 2009. Seismic evidence for overpressured subducted oceanic crust and megathrust fault sealing, Nature, 457, 76-78.

Balfour, N., Savage, M. \& Townend, J., 2005. Stress and crustal anisotropy in Marlborough, New Zealand: evidence for low fault strength and structurecontrolled anisotropy, Geophys. J. Int., 163(3), 1073-1086.

Bernal-López, L.A., Garibaldi, B.R., León Soto, G., Valenzuela, R.W. \& Escudero, C.R., 2016. Seismic anisotropy and mantle flow driven by the Cocos slab under southern Mexico, Pure appl. Geophys., 173, 3373-3393.

Bezacier, L., Reynard, B., Bass, J.D., Sanchez-Valle, C. \& Van de Moortele, B., 2010. Elasticity of antigorite, seismic detection of serpentinites and anisotropy in subduction zones, Earth planet Sci. Lett., 289, 198-208.

Blatter, D.L. \& Hammersley, L., 2010. Impact of the Orozco Fracture Zone on the central Mexican Volcanic Belt, J. Volc. Geotherm. Res., 197, 67-84.

Boness, N.L. \& Zoback, M.D., 2004. Stress-induced seismic velocity anisotropy and physical properties in the SAFOD Pilot Hole in Parkfield, California, Geophys. Res. Lett., 31, L15S17, doi:10.1029/2003GL019020.

Boness, N.L. \& Zoback, M.D., 2006. A multiscale study of the mechanisms controlling shear velocity anisotropy in the San Andreas Fault Observatory at depth, Geophysics, 71(5), F131-F146.

Bowman, J.R. \& Ando, M., 1987. Shear wave splitting in the upper mantle wedge above the Tonga subduction zone, Geophys. J. R. astr. Soc., 88, $25-41$.

Cassidy, J.F., 1992. Numerical experiments in broadband receiver function analysis, Bull. seism. Soc. Am., 82, 1453-1474.

Cochran, E., Li, Y.-G. \& Vidale, J., 2006. Anisotropy in the shallow crust observed around the San Andreas Fault before and after the 2004 M 6.0 Parkfield earthquake, Bull. seism. Soc. Am., 96, S364-S375.

Chen, T. \& Clayton, R.W., 2009. Seismic attenuation structure in central Mexico: image of a focused high-attenuation zone in the mantle wedge, J. geophys. Res., 114, B07304, doi:10.1029/2008JB005964.

Chen, T. \& Clayton, R.W., 2012. Structure of central and southern Mexico from velocity and attenuation tomography, J. geophys. Res., 117, B09302, doi:10.1029/2012JB009233.

Chen, Y. \& Niu, F., 2013. Ray-parameter based stacking and enhanced preconditioning for stable inversion of receiver function data, Geophys. $J$. Int., 194, 1682-1700.

Chevrot, S. \& Van der Hilst, R.D., 2000. The Poisson's ratio of the Australian crust: geological and geophysical implications, Earth planet. Sci. Lett., 183, 121-132.

Christensen, N.I., 1984. The magnitude, symmetry and origin of upper mantle anisotropy based on fabric analyses of ultramafic tectonics, Geophys. J. R. astr. Soc., 76, 89-111.

Clayton, R.W. \& Wiggins, R.A., 1976. Source shape estimation and deconvolution of teleseismic bodywaves, Geophys. J. R. astr. Soc., 47, 151-177.

Crampin, S., 1990. The scattering of shear waves in the crust, Pure appl. Geophys., 132, 67-91.

Crampin, S., 1994. The fracture criticality of crustal rocks, Geophys. J. Int., 118, 428-438.

Crampin, S. \& Chastin, S., 2003. A review of shear-wave splitting in the crack-critical crust, Geophys. J. Int., 155, 221-240.
Crampin, S. \& Gao, Y., 2006. A review of techniques for measuring shearwave splitting above small earthquakes, Phys. Earth planet. Inter, 159, $1-14$.

Crampin, S. \& Zatsepin, S.V., 1997. Modelling the compliance of crustal rock. II. Response to temporal changes before earthquakes, Geophys. J. Int., 129, 495-506.

DeMets, C. \& Wilson, D.S., 1997. Relative motions of the Pacific, Rivera, North American and Cocos plates since 0.78 Ma, J. geophys. Res., 102, 2789-2806.

Dougherty, S.L. \& Clayton, R.W., 2014. Seismicity and structure in central Mexico: evidence for a possible slab tear in the South Cocos plate, $J$. geophys. Res., 119, 3424-3447.

Dougherty, S., Clayton, R. \& Helmberger, D., 2012. Seismic structure in Central Mexico: implication for fragmentation of the subducted Cocos plate, J. geophys. Res., 117, B09316, doi:10.1029/2012JB009528.

Efron, E. \& Tibshirani, R.J., 1993. An Introduction to the Bootsrap, Chapman \& Hall, New York.

Ferrari, L., Orozco-Esquivel, T., Manea, V.C. \& Manea, M., 2012. The dynamic history of the Trans-Mexican Volcanic Beltandthe Mexico subduction zone, Tectonophysics, 522-523, 122-149.

Frederiksen, A.W. \& Bostock, M.G., 2000. Modelling teleseismic waves in dipping anisotropic structures. Geophys. J. Int., 141, 401-412.

Gilje, E. \& Thomsen, I., 1970. Two methods for splitting data into homogeneous groups, Stat. Rev., 8(4), 307-314.

Gill, J.B., 1981. Orogenic andesites and Plate Tectonics, Minerals and Rocks, Vol. 16, Springer, Berlin.

Gomez-Tuena, A., Orozco-Esquivel, T. \& Ferrari, L., 2007. Igneous petrogenesis of the Trans-Mexican Volcanic belt. Geol. Soc. Am. Spec. Pap., 442, 129-181.

Greene Gondi, F., 2009. Geometría de la placa de Cocos usando funciones de receptor, MSc thesis, Posgrado en Ciencias de la Tierra, Universidad Nacional Autónoma de México, Mexico City, Mexico, p. 118.

Helffrich, G. \& Wood, B.J., 2001. The Earth's mantle, Nature, 412, 501-507.

Hudson, J.A., 1994. Overall properties of anisotropic materials containing cracks, Geophys. J. Int., 116, 279-282.

Huesca-Pérez, E. \& Husker, A., 2012. Shallow travel-time tomography bellow Southern Mexico, Geofis. Int., 51, 281-291.

Huesca-Pérez, E., Valenzuela, R.W. \& Ortega, R., 2016. Crustal anisotropy from tectonic tremor in Guerrero, Mexico, Geochem. Geophys. Geosyst., 17, 2323-2335.

Husker, A. \& Davis, P.M., 2009. Tomography and thermal state of the Cocos plate subduction beneath Mexico City, J. geophys. Res., 114, B04306, doi:10.1029/2008JB006039.

Iglesias, A., Clayton, R.W., Pérez-Campos, X., Singh, S.K., Pacheco, J.F., García, D. \& Valdés-González, C., 2010. S wave velocity structure below central Mexico using high-resolution surface wave tomography, J. geophys. Res., 115, B06307, doi:10.1029/2009JB006332.

Jödicke, H., Jording, A., Ferrari, L., Arzate, J., Mezger, K. \& Rüpke, L., 2006. Fluid release from the subducted Cocos plate and partial melting of the crust deduced from magnetotelluric studies in southern Mexico: implications for the generation of volcanism and subduction dynamics, J. geophys. Res., 111, B08102, doi:10.1029/2005JB003739.

Jung, H. \& Karato, S.I., 2001. Water-induced fabric transitions in olivine, Science, 293, 1460-1463.

Kaneshima, S., 1990. Origin of crustal anisotropy: shear wave splitting studies in Japan, J. geophys. Res., 95, 11 121-11 133.

Karato, S.I., Jung, H., Katayama, I. \& Skemer, P., 2008. Geodynamic significance of seismic anisotropy of the upper mantle: new insights from laboratory studies, Annu. Rev. Earth planet. Sci., 36, $59-95$.

Kim, Y., Miller, M.S., Pearce, F. \& Clayton, R.W., 2012. Seismic imaging of the Cocos plate subduction zone system in central Mexico, Geochem. Geophys. Geosyst., 13, Q07001, doi:10.1029/2012GC004033.

Kim, Y., Clayton, R.W. \& Jackson, J.M., 2010. Geometry and seismic properties of the subducting Cocos plate in central Mexico, J. geophys. Res., 115, B06310, doi:10.1029/2009JB006942. 
Kim, Y., Clayton, R.W., Asimow, P.D. \& Jackson, J.M., 2013. Generation of talc in the mantle wedgeandits role in subduction dynamics in central Mexico, Earth planet. Sci. Lett., 384, 81-87.

Kneller, E.A., van Keken, P.E., Karato, S.-I. \& Park, J., 2005. B-type olivine fabric in the mantle wedge: insights from high-resolution nonNewtonian subduction zone models, Earth planet. Sci. Lett., 237, 781-797.

Kodaira, S., Iidaka, T., Kato, A., Iwasaki, T. \& Kaneda, Y., 2004. High pore fluid pressure may cause silent slip in the Nankai Trough, Science, 304, $1295-1298$.

Langston, C.A., 1977. The effect of planar dipping structure on source and receiver responses for constant ray parameter, Bull. seism. Soc. Am., 67, 1029-1050.

Langston, C.A., 1979. Structure under Mount Rainier, Washington, inferred from teleseismic body waves, J. geophys. Res., 84, 4749-4762.

Langston, C.A., 1989. Scattering of teleseismic body waves under Pasadena, California, J. geophys. Res., 94, 1935-1951.

León Soto, G., Ni, J.F., Grand, S.P., Sandvol, E., Valenzuela, R.W., Guzmán Speziale, M., Gómez González, J.M. \& Domínguez Reyes, T., 2009. Mantle flow in the Rivera-Cocos subduction zone, Geophys. J. Int., 179, 1004-1012.

Levin, V. \& Park, J., 1998. P-SH conversions in layered media with hexagonally symmetric anisotropy: a cookbook, in Geodynamics of Lithosphere and Earth's Mantle, Pageoph Topical Volumes, pp. 669-697, eds Plomerová, J., Liebermann, R.C. \& Babuška, V., Birkhäuser Basel.

Long, M.D., 2009a. Geophysics: going with the mantle flow, Nat. Geosci., 2, $10-11$.

Long, M.D., 2009b. Complex anisotropy in D" beneath the eastern Pacific from SKS-SKKS splitting discrepancies, Earth planet. Sci. Lett., 283, 181-189.

Long, M.D. \& Silver, P.G., 2008. The subduction zone flow field from seismic anisotropy: a global view, Science, 319, 315-318.

Mainprice, D. \& Ildefonse, B., 2009. Seismic anisotropy of subduction zone minerals - contribution of hydrous phases, in Frontiers in Earth Sciences: Subduction Zone Geodynamics, pp. 63-84, eds Plomerová, J., Liebermann, R.C. \& Babuška, V., Springer.

Mainprice, D. \& Nicolas, A., 1989. Development of shape and lattice preferred orientation: application to the seismic anisotropy of the lower crust, J. Struct. Geol., 11, 175-189.

Manea, V., Manea, M., Kostoglodov, V. \& Sewell, G., 2006. Intraslab seismicity and thermal stress in the subducted Cocos plate beneath Central Mexico, Tectonophysics, 420, 389-408.

Manea, M., Manea, V., Ferrari, L., Kostoglodov, V. \& Bandy, W., 2005. Tectonic evolution of the Tehuantepec Ridge, Earth planet. Sci. Lett., 238, 64-77.

Manea, V.C., Manea, M. \& Ferrari, L., 2013. A geodynamical perspective on the subduction of Cocos and Rivera plates beneath Mexico and Central America, Tectonophysics, 609, 56-81.

MASE, 2007. Meso America subduction experiment, Caltech. Dataset, doi:10.7909/C3RN35SP.

Mavko, G., Mukerji, T. \& Dvorkin, J., 1998. The Rock Physics Handbook, Cambridge Univ. Press.

McNamara, D.E. \& Owens, T.J., 1993. Azimuthal shear wave velocity anisotropy in the Basin and Range province using Moho Ps converted phases, J. geophys. Res., 98, 12 003-12 017.

Melgar, D. \& Pérez-Campos, X., 2011. Imaging the Moho and subducted oceanic crust at the isthmus of Tehuantepec, Mexico, from receiver functions, Pure appl. Geophys., 168, 1449-1460.

Nair, S.K., Gao, S.S., Liu, K.H. \& Silver, P.G., 2006. Southern African curstal evolution and composition: constraints from receiver function studies, J. geophys. Res., 111, B02304, doi:10.1029/2005JB003802.

Nicolas, A. \& Christensen, N.I., 1987. Formation of anisotropy in upper mantle peridotites - a review, in the composition, structure and dynamics of the lithosphere-asthenosphere system, Am. geophys. Un. Geodyn. Ser., 16, 111-123.

Pardo, M. \& Suárez, G., 1995. Shape of the subducted Rivera and Cocos plates in southern Mexico: seismic and tectonic implications, J. geophys. Res., 100, 12 357-12 373 .
Peacock, S.M., Christensen, N.I., Bostock, M.G. \& Audet, P., 2011. High pore pressuresandporosity at $35 \mathrm{~km}$ depth in the Cascadia subduction zone, Geology, 39, 471-475.

Pérez-Campos, X. et al., 2008. Horizontal subduction and truncation of the Cocos plate beneath central Mexico, Geophys. Res. Lett., 35, L18303, doi:10.1029/2008GL035127.

Ponce Cortés, J.G., 2012. Medición de la anisotropía de las ondas SKS en el manto superior, debajo de las estaciones permanentes del Servicio Sismológico Nacional instaladas a partir del año 2005, BSc thesis, Facultad de Ingeniería, Universidad Nacional Autónoma de México, Mexico City, Mexico, p. 79.

Randall, G.E. \& Owens, T.J., 1994. Array analysis of teleseismic Pwaveforms from the 1988-89 PASSCAL, Basin and Range passive source seismic experiment, Geophys. J. Int., 116, 618-636.

Rojo Garibaldi, B., 2011. Anisotropía de las ondas SKS en el manto superior debajo de un arreglo sísmico entre Guerrero y Veracruz, BSc thesis, Facultad de Ciencias, Universidad Nacional Autónoma de México, Mexico City, Mexico, p. 85.

Sandvol, E., Seber, D., Barazangi, M., Vernon, F., Mellors, R. \& Al-Amri, A., 1998. Lithospheric seismic velocity discontinuities beneath the Arabian Shield, Geophys. Res. Lett., 25, 2873-2876.

Savage, M.K., 1998. Lower crustal anisotropy or dipping boundaries? Effects on receiver functions and a case study in New Zealand, J. geophys. Res., 103, 15069-15087.

Savage, M.K., 1999. Seismic anisotropy and mantle deformation: what have we learned from shear wave splitting?, Rev. Geophys., 37, 65-106.

Savage, M.K., Peppin, W.A. \& Vetter, U.R., 1990. Shear-wave anisotropyandstress direction in and near Long Valley Caldera, Califoria, 19791988, J. geophys. Res., 95, 11 165-11 177.

Silver, P.G., 1996. Seismic anisotropy beneath the continents: probing the depths of Geology, Annu. Rev. Earth planet. Sci., 24, 385-432.

Silver, P.G. \& Chan, W.W., 1988. Implications for continental structure andevolution from seismic anisotropy, Nature, 335, 34-39.

Silver, P.G. \& Chan, W.W., 1991. Shear wave splitting and subcontinental mantle deformation, J. geophys. Res., 96, 16 429-16 454.

Singh, S.K. \& Pardo, M., 1993. Geometry of the Benioff zone and state of stress in the overriding plate in central Mexico, Geophys. Res. Lett., 20(14), 1483-1486

Solari, L.A., Torres de León, R., Hernández Pineda, G., Solé, J. \& SolísPichardo, G., 2007. Tectonic significance of Cretaceous-Tertiary magmatic and structural evolution of the northern margin of the Xolapa Complex, Tierra Colorada area, southern Mexico, Geol. Soc. Am. Bull., 119, $1265-1279$.

Song, T.-R. \& Kim, Y., 2012a. Anisotropic uppermost mantle in young subducted slab underplating central Mexico, Nat. Geosci., 5, 55-59.

Song, T.-R. \& Kim, Y., 2012b. Localized seismic anisotropy associated with long-term slow-slip events beneath southern Mexico, Geophys. Res. Lett., 39, L09308, doi:10.1029/2012GL051324.

Song, T.-R.A., Helmberger, D.V., Brudzinski, M.R., Clayton, R.W., Davis, P., Pérez-Campos, X. \& Singh, S.K., 2009. Subducting slab ultra-slow velocity layer coincident with silent earthquakes in southern Mexico, Science, 324, 502-506.

Stubailo, I., Beghein, C. \& Davis, P.M., 2012. Structure and anisotropy of the Mexico subduction zone based on Rayleigh-wave analysis and implocations for the geometry of the Trans-Mexican Volcanic Belt, $J$. geophys. Res., 117, B05303, doi:10.1029/2011JB008631.

Suárez, G. \& Singh, S.K., 1986. Tectonic interpretation of the TransMexican Volcanic Belt-discussion, Tectonophysics, 127, 155-158.

Suter, M., 1991. State of stress and active deformation in Mexico and western Central America, in Neotectonics of North America, pp. 401-421, Geological Society of America Memoir, doi:10.1130/dnagcsms-neo.

Valenzuela, R.W. \& León Soto, G., 2017. Shear wave splittingandmantle flow in Mexico: what have we learned?, Geofisica Internacional, 56, 187-217.

van Benthem, S.A.C., Valenzuela, R.W. \& Ponce, G.J., 2013. Measurements of upper mantle shear wave anisotropy from a permanent network in southern Mexico, Geofis. Int., 52, 385-402. 
Villafuerte, C., 2014. Modelado poroelástico de sismos silenciosos en Guerrero, México, BSc thesis, Facultad de Ingeniería, Universidad Nacional Autónoma de México, Mexico City, Mexico, p. 123.

Vinnik, L., Chevrot, S. \& Montagner, J.P., 1997. Evidence for a stagnant plume in the transition zone?, Geophys. Res. Lett., 24, 1007-1010.

Wang, X.-Q., Schubnel, A., Fortin, J., David, E.C., Guéguen, Y. \& Ge, H.-K., 2012. High Vp Vs ratio: saturated cracks or anisotropy effects?, Geophys. Res. Lett., 39, L11307, doi:10.1029/2012GL051742.

Zhang, S. \& Karato, S.-I., 1995. Lattice preferred orientation of olivine aggregates deformed in simple shear, Nature, 375, 774-777.

Zhu, L. \& Kanamori, H., 2000. Moho depth variations in Southern California from teleseismic receiver functions, J. geophys. Res., 105, 2969-2980.

\section{SUPPORTING INFORMATION}

Supplementary data are available at $G J I$ online.

\section{Appendix A: Anisotropic component characterization for non- horizontal layers}

Figure A1. Radial and tangential RFs of station PLAY (left). The colour of the RFs indicates its backazimuth geographic quadrant (light blue is NE, blue is SE, green is SW and red is NW). The dashed lines over the RFs represent the continental crust (green), upper oceanic crust (blue) and lower oceanic crust (red) pulses. Characterization process of the timing variations of the analysed Ps phases (right). The colour format is the same as in the left figure. The fitted curve parameters were obtained using a grid search algorithm for all coherent values of $A, T, \varphi$ and $C$. The $\varphi$ values for the analysed phases of this station are: $205^{\circ}, 157^{\circ}$ and $183^{\circ}$, progressively.

Figure A2. Example of used and discarded RFs rotated to the fast/slow system from which reliable measurements of $\delta t$ were obtained for the different $P S$ phases analysed in station PLAY. The dashed red lines represent the splitting between the two phases.

\section{Appendix B: Anisotropy parameters}

Table B1. Average anisotropy parameters for the continental crust. Table B2. Average anisotropy parameters for the upper oceanic crust.

Table B3. Average anisotropy parameters for the lower oceanic crust.

Table B4. Anisotropy parameters for the upper mantle.

Please note: Oxford University Press is not responsible for the content or functionality of any supporting materials supplied by the authors. Any queries (other than missing material) should be directed to the corresponding author for the paper. 